\title{
Low bit-rate Video Transmission over Wireless Zigbee Networks
}

\author{
by
}

\author{
AHMED HAFIZ ZAINALDIN \\ A thesis submitted to \\ the Faculty of Graduate Studies and Research \\ in partial fulfilment of \\ the requirements for the degree of \\ Master of Applied Science in Electrical Engineering \\ Ottawa-Carleton Institute for \\ Electrical and Computer Engineering \\ Department of Systems and Computer Engineering \\ Carleton University \\ Ottawa, Ontario, Canada \\ December 2007
}

Copyright (C)

2007 - AHMED HAFIZ ZAINALDIN 


$\begin{array}{ll}\begin{array}{l}\text { Library and } \\ \text { Archives Canada }\end{array} & \begin{array}{l}\text { Bibliothèque et } \\ \text { Archives Canada }\end{array} \\ \begin{array}{l}\text { Published Heritage } \\ \text { Branch }\end{array} & \begin{array}{l}\text { Direction du } \\ \text { Patrimoine de l'édition }\end{array} \\ \begin{array}{l}\text { 395 Wellington Street } \\ \text { Ottawa ON K1A 0N4 } \\ \text { Canada }\end{array} & \begin{array}{l}\text { 395, rue Wellington } \\ \text { Ottawa ON K1A ON4 }\end{array} \\ \text { Canada }\end{array}$

Your file Votre référence ISBN: 978-0-494-36837-4 Ourfile Notre référence ISBN: 978-0-494-36837-4

\section{NOTICE:}

The author has granted a nonexclusive license allowing Library and Archives Canada to reproduce, publish, archive, preserve, conserve, communicate to the public by telecommunication or on the Internet, loan, distribute and sell theses worldwide, for commercial or noncommercial purposes, in microform, paper, electronic and/or any other formats.

The author retains copyright ownership and moral rights in this thesis. Neither the thesis nor substantial extracts from it may be printed or otherwise reproduced without the author's permission.
AVIS:

L'auteur a accordé une licence non exclusive permettant à la Bibliothèque et Archives Canada de reproduire, publier, archiver, sauvegarder, conserver, transmettre au public par télécommunication ou par l'Internet, prêter, distribuer et vendre des thèses partout dans le monde, à des fins commerciales ou autres, sur support microforme, papier, électronique et/ou autres formats.

L'auteur conserve la propriété du droit d'auteur et des droits moraux qui protège cette thèse. $\mathrm{Ni}$ la thèse ni des extraits substantiels de celle-ci ne doivent être imprimés ou autrement reproduits sans son autorisation.
In compliance with the Canadian Privacy Act some supporting forms may have been removed from this thesis.

While these forms may be included in the document page count, their removal does not represent any loss of content from the thesis.
Conformément à la loi canadienne sur la protection de la vie privée, quelques formulaires secondaires ont été enlevés de cette thèse.

Bien que ces formulaires aient inclus dans la pagination, il n'y aura aucun contenu manquant.

\section{Canadä}


The undersigned recommends to

the Faculty of Graduate Studies and Research

acceptance of the thesis

\title{
Low bit-rate Video Transmission over Wireless Zigbee Networks
}

\author{
Submitted by AHMED HAFIZ ZAINALDIN \\ in partial fulfilment of the requirements for the degree of \\ Master of Applied Science in Electrical Engineering
}

Ioannis Lambadaris, Co-Supervisor

Biswajit Nandy, Co-Supervisor

Victor C. Aitken, Department Chair

Carleton University

2007 


\section{Abstract}

The emerging IEEE 802.15.4 (Zigbee) standard is designed for low data rate, low power consumption and low cost wireless personal area networks (WPANs). Video transmission over such networks is considered an issue since video traffic demands high data rates.

Video surveillance applications can be used for cost effective deployments. However, for an acceptable video quality, the transmission of video over such networks is considered an issue since video traffic contains a large amount of information that requires high data rates. In this thesis, the performance of a video surveillance application is analyzed over wireless Zigbee networks using ns-2 simulations.

The Transform-Expand-Sample (TES) methodology is used to model a low datarate MPEG4 video. A rate control variable bit-rate algorithm (RC-VBR) adapted to MPEG4 variable bit-rate (VBR) video coders is developed over Zigbee networks. A region-of-interest (ROI) encoding is added to the rate control algorithm to capture the important parts of the frame which is suitable for IEEE 802.15.4 (Zigbee) networks.

The IEEE 802.15.4 network can improve the aggregate throughput by using multiple channels that are available in the radio spectrum allocated by the standard. A multi-channel over IEEE 802.15.4 network architecture is investigated by equipping each Zigbee node with multiple 802.15.4 network interface cards (NICs).

A Zigbee network will enable a large number of surveillance applications to operate over a personal area network. 


\section{Acknowledgments}

I would like to express my deep-felt gratitude to my advisors, Prof. Ioannis Lambadaris and Prof. Biswajit Nandy of the Systems and Computer Engineering Department at Carleton University, for their guidance, advice, encouragement, enduring patience and constant support during the writing of this thesis. I would also like to thank them for providing me with clear explanations when needed, and for constantly encouraging me when facing any obstacles.

I would also like to thank everyone who contributed in any way to help me during this thesis. Special thanks to all the members of my family, my father, mother, brothers and my sister. 


\section{Table of Contents}

Abstract

Acknowledgments iv

Table of Contents $\quad$ v

List of Tables $\quad$ viii

List of Figures $\quad$ ix

List of Abbreviations $\quad$ xii

1 Introduction 1

1.1 Background and Motivation .................. 1

1.2 Objective of the Thesis . . . . . . . . . . . . . . 4

1.3 Thesis Outline ..................... 5

1.4 Thesis Contributions . . . . . . . . . . . . . . . 6

2 Overview of Wireless Zigbee Networks and Video Encoding $\quad 8$

2.1 Wireless Zigbee networks . . . . . . . . . . . . . . 8

$2.1 .1 \quad$ ZigBee Alliance ................. 8

2.1.2 Origins of the name ZigBee .............. 9

2.1.3 Overview of the IEEE 802.15 .4 standard . . . . . . . . . 9 
2.1.4 IEEE 802.15.4 Interference . . . . . . . . . . . . 15

2.2 Overview of video encoding standards . . . . . . . . . . . 18

2.2.1 Constant bit-rate (CBR) coding . . . . . . . . . 18

2.2.2 Variable bit-rate (VBR) coding . . . . . . . . . 19

2.2.3 Overview of the MPEG4 video encoding standard . . . . . . 20

2.3 Multimedia applications over wireless Zigbee and sensor networks . . 23

3 Introduction to Video Traffic Modeling using Transform-ExpandSample (TES) Processes $\quad 26$

3.1 Overview of Transform-Expand-Sample (TES) processes . . . . . . 28

3.2 TES modeling of MPEG4 video . . . . . . . . . . . . 30

3.2.1 Verification of TES modeling scaling factor of MPEG4 video

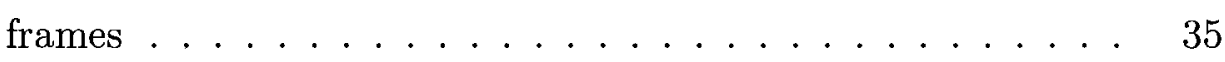

4 Rate Control Variable bit-rate (RC-VBR) over Wireless Zigbee $\begin{array}{ll}\text { Networks } & 39\end{array}$

4.1 Rate Control Video Transmission . . . . . . . . . . . . . . . 40

4.2 Rate control variable bit-rate (RC-VBR) algorithm over wireless Zigbee

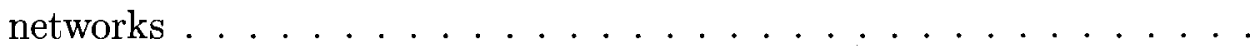

4.3 Region-of-interest based rate control variable bit-rate (ROI RC-VBR) algorithm over wireless Zigbee networks . . . . . . . . . . . . 44

5 Multi-channel Multi-radio over Wireless Zigbee Networks 46

5.1 Related work of multi-channel multi-radio network topology . . . . . 47

5.2 Extension for multi-channel multi-radio over IEEE 802.15.4/Zigbee

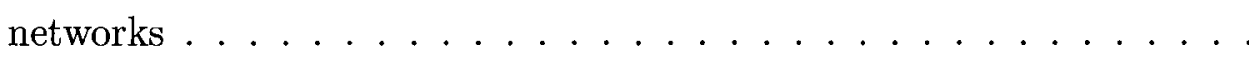

5.3 Multiple Description Coding (MDC) over a multi-channel multiinterface Zigbee networks . . . . . . . . . . . . . . . . . . . . . . . 50 
5.3.1 Multiple Layer Coding (MLC) . . . . . . . . . . . . . 51

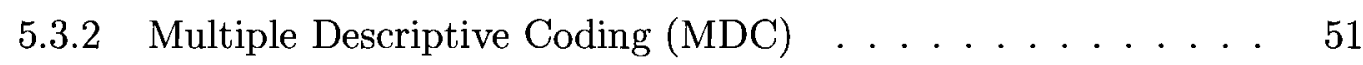

5.3.3 Related Studies of multiple coding mechanisms . . . . . . 51

\section{Experiments using Simulation Results of Wireless Zigbee Networks 54}

6.1 Validity of TES models over wireless Zigbee networks using simulation 56

6.2 Simulation results of RC-VBR algorithm over wireless Zigbee networks 58

6.3 Simulation results of ROI RC-VBR algorithm over wireless Zigbee networks . . . . . . . . . . . . . . . . . . . . 64

6.4 Effects of interference of WLANs (IEEE 802.11) and LR-WPANs (IEEE 802.15.4/Zigbee) on video surveillance LR-WPANs $\ldots \ldots .68$

6.5 Simulation study of multi-channel multi-radio over wireless Zigbee net-

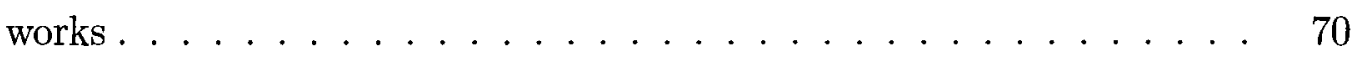

6.6 Network topology and simulations of Multiple Description Coding (MDC) over multi-radio multi-channel Zigbee networks . . . . . . 73

7 Conclusions and Future Work $\quad 78$

7.1 TES modeling for low bit-rate MEPG4 video and future directions . . 78

7.2 Summary of implemented topic results and future work . . . . . . 79

7.3 Low power consumption and low complexity video compression over wireless Zigbee networks . . . . . . . . . . . . . 81

7.4 Scheduler design of multi-channel multi-interface topology $\ldots \ldots .83$

7.5 Enhanced channel modeling for simulations . . . . . . . . . . . . 84 


\section{List of Tables}

3.1 Statistics and parameters of the models . . . . . . . . . . . 35

6.1 PSNR to MOS mapping . . . . . . . . . . . . . 55

6.2 Simulation Parameters .................. 61

6.3 Comparison of the performance metrics of VBR and RC-VBR algorithms 63

6.4 Comparison of the performance metrics of ROI-VBR, RCVBR and ROI-RCVBR algorithms with 4 video sources . . . . . . . . . 66

6.5 Impact of interference on IEEE 802.15 .4 networks . . . . . . . . . . . 69

6.6 Effect of number of sources on a 2 channel and a 2 interface topology 71

6.7 Effect of number of sources on a 3 channel and a 2 interface topology 72

6.8 Comparison of the performance metrics of ROI-RCVBR with and with-

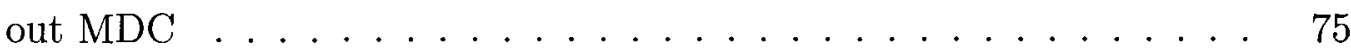




\section{List of Figures}

2.1 Star and peer-to-peer topology examples . . . . . . . . . . 11

2.2 Zigbee OSI Layers . . . . . . . . . . . . . . . . . . . 12

2.3 LR-WPAN and WLAN interferece . . . . . . . . . . . 16

2.4 Frame Index vs. Quantization Parameter . . . . . . . . . . . . 19

2.5 MPEG4 Overview ....................... 21

2.6 MPEG4 frame sequences. . . . . . . . . . . . . . . . 22

3.1 Empirical video frame trace with $\mathrm{Q}=7 \ldots \ldots 31$

3.2 Empirical histogram with $\mathrm{Q}=7 \ldots \ldots 32$

3.3 Empirical autocorrelation function with $\mathrm{Q}=7 \ldots \ldots 32$

3.4 Original and generated model of I-frames . . . . . . . . . . . . 33

3.5 Original and generated model of the I-frames histogram . . . . . . . . 33

3.6 Original and generated model of the I-frames autocorrelation function 34

3.7 Innovation function of the I-frames model f . . . . . . . . . . 34

3.8 Modeled vs. Real TES data for the I frames as appears in the testool 35

3.9 Modeled vs. Real TES data for the P frames as appears in the testool 36

3.10 Modeled vs. Real TES data for the B frames as appears in the testool 37

3.11 Modeled vs. Real TES data for the overall frame sequence as appears

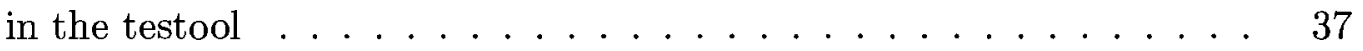

3.12 Frame sequence statistics as appears in the testool for Q 7 and $12 \ldots 38$

3.13 Scaling Factor vs. Quantization parameter . . . . . . . . . . 38 
$4.1 \quad$ RC-VBR encoder . . . . . . . . . . . . . . . . . . 41

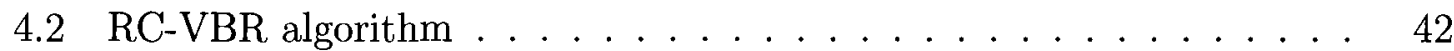

4.3 GOP Index vs. R-Q Product . . . . . . . . . . . . . . . . . 43

4.4 a) Real video frame sample b) Video frame using video segmentation 44

5.1 Ns Node Structure . . . . . . . . . . . . . . . . . . . . 53

6.1 a) One hop Zigbee video transmission. b) Two hop Zigbee video trans-

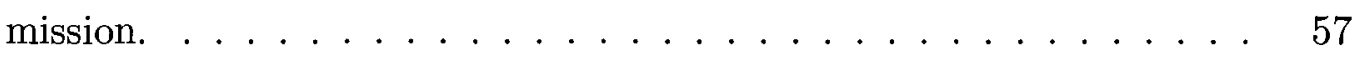

6.2 Frame Loss vs. Buffer size (bytes) . . . . . . . . . . . . . . 58

6.3 Mean Delay(sec) vs. Quantization Parameter . . . . . . . . . . . . 59

6.4 Network Topology of the RC-VBR algorithm . . . . . . . . . . . 59

6.5 Number of hops vs. Throughput (packets/sec) . . . . . . . . . 60

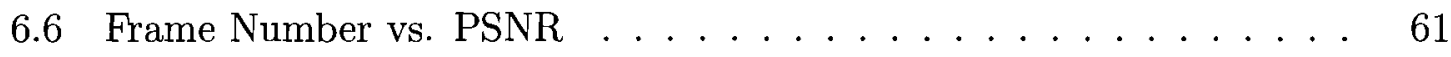

6.7 Comparison of i)Real video ii)RC-VBR algorithm and iii)VBR encoded sample frames . . . . . . . . . . . . . . . 62

6.8 i) Real video ii) RC-VBR algorithm iii) VBR encoded sample frames 64

6.9 Video bit-rate vs. Frame Loss . . . . . . . . . . . . . . . 65

6.10 Video bit-rate vs. Average PSNR . . . . . . . . . . . . 65

6.11 LR-WPAN and WLAN interference topologies $\ldots \ldots \ldots \ldots$

6.12 Network Topology with 2 channels and 2 interfaces . . . . . . . 70

6.13 Network Topology with 3 channels and 2 interfaces . . . . . . . 72

6.14 Network Topology with 4 channels and 2 interfaces with MDC . . . 73

6.15 Multiple Descriptive Coding (MDC) . . . . . . . . . . . . 74

6.16 Gilbert-Eliot error model . . . . . . . . . . . . . . . . 75

6.17 Comparison of multi-channel multi-interface using i) with MDC and

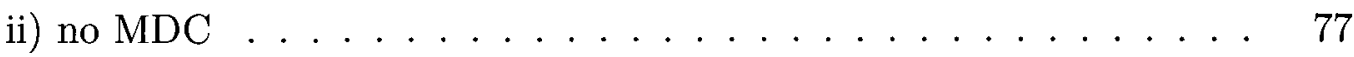

7.1 Simple compression algorithm for wireless surveillance Zigbee networks 83

7.2 Scheduler of a multi-channel multi-interface 802.15 .4 node $\ldots . . \quad 85$ 
7.3 FSMC model for IEEE 802.15 .4 channels $\ldots \ldots \ldots \ldots \ldots \ldots$ 


\section{List of Abbreviations}

$\begin{array}{ll}\text { ACK } & \text { Acknowledgment } \\ \text { AODV } & \text { Ad hoc On Demand Distance Vector } \\ \text { ARQ } & \text { Automatic Repeat Request } \\ \text { CAP } & \text { Contention access period } \\ \text { CBR } & \text { Constant-bit-rate coding } \\ \text { CCA } & \text { Clear Channel Assessment } \\ \text { CFP } & \text { Contention free period } \\ \text { CSMA-CA } & \text { Carrier Sense Multiple Access- Collision Avoidance } \\ \text { DSSS } & \text { Direct Sequence Spread Spectrum } \\ \text { FEC } & \text { Forward Error Correction } \\ \text { FFD } & \text { Full Function Device } \\ \text { GOB } & \text { Group of Blocks } \\ \text { GOP } & \text { Group of Pictures } \\ \text { GOV } & \text { Group of VOPs }\end{array}$




$\begin{array}{ll}\text { GTS } & \text { Guaranteed Time Slot } \\ \text { ISO } & \text { International Organization for Standardization } \\ \text { JVT } & \text { Joint Video Team } \\ \text { LR-WPAN } & \text { Low Rate Wireless Personal Area Networks } \\ \text { MAC } & \text { Media Access Controller } \\ \text { M-JPEG } & \text { Motion-JPEG } \\ \text { MPEG } & \text { Moving Picture Expert Group } \\ \text { MSE } & \text { Mean Square Error } \\ \text { O-QPSK } & \text { Offset Quadrature Phase Shift Keying } \\ \text { OSI } & \text { Open System Interconnection } \\ \text { PAN } & \text { Personal Area Network } \\ \text { PSDU } & \text { PHY Service Data Unit } \\ \text { PSNR } & \text { Peak Signal to Noise Ratio } \\ \text { RC-VBR } & \text { Rate control variable-bit-rate coding } \\ \text { RFD } & \text { Reduced Function Device } \\ \text { ROI } & \text { Region of interest } \\ \text { TES } & \text { Transmission Control Protocol } \\ \text { Transform-Expand-Sample } \\ \text { RE }\end{array}$


UDP User Datagram Protocol

VBR Variable-bit-rate coding

VO Video Object

VOP Video Object Plane

WSN Wireless Sensor Network

ZC ZigBee Coordinator

ZED ZigBee End Device

ZR ZigBee Router 


\section{Chapter 1}

\section{Introduction}

\subsection{Background and Motivation}

Most of the attention on wireless standards, i.e. IEEE 802.11 and cellular networks, focus on high rate and long range applications. The concept of wireless personal area networks (WPANs) has been around for several years, entering the mainstream with the creation of the IEEE 802.15 Working Group in 1997. This large and diverse community of technologists, scientists, and visionaries came together under the IEEE Standards Association (IEEE-SA) to share their views on short-range wireless networking where little or no infrastructure is required (no network setups and no access points). The dream was ubiquitous, un-tethered, short-range communications.

The first vision was to design a technology centered on cable replacement for consumer electronic devices which was called Bluetooth (IEEE Std 802.15.1). The second vision (IEEE Std 802.15.3) focused on high-speed WPANs driven by the need for wireless connectivity in multimedia devices. In 2000, the IEEE-SA New Stan-

dards Commtttee (NesCom) officially sanctioned the creation of a new project to begin development of a third standard (IEEE Std 802.15.4 [1]) for low-rate wireless personal area networks (LR-WPANs). The goal of the IEEE 802.15.4 standard was 
to provide ultra-low complexity, low cost and extremely low-power wireless connectivity among inexpensive fixed, portable, and moving devices. Simplicity was the watchword throughout the standard development process. The IEEE 802.15.4 standard is thus designed to address applications with relaxed throughput and latency requirements while favoring a low-cost and low-power design. These features make possible, applications in various industries such as agriculture, residential security, remote diagnostics, home automation, personal healthcare and surveillance. Wireless video surveillance will be the focus of this thesis.

There are various applications for wireless video surveillance networks such as theft detection, health monitoring and battle field reconnaissance within the military.

In general, IEEE 802.11, Bluetooth and other technologies are more suitable for real time applications than IEEE 802.15.4 since they offer much higher data rates. However, the cost of video transmission will be lowered if IEEE 802.15.4 based devices are used. A number of reasons enable video to be transmitted over low data-rate networks. Some of them being advanced video compression techniques such as MPEG4 and H.264, advanced content based video segmentation and rate control algorithms. Wireless Zigbee networks [10] are low data rate networks that implement the IEEE 802.15.4 as their physical and MAC layers. It is believed that wireless Zigbee networks may become the most used standard for wireless sensor networks (WSNs).

Wireless Zigbee and sensor networks substantially reduce the cost of installation and deployment in industrial facilities. More deployment means better visibility into operational and environmental variables that affect overall safety, and compliance.

ZigBee's RF power specification comes from IEEE 802.15.4, which specifies a minimum power output rating of $1 \mathrm{~mW}$, with no specified maximum power. The communication range is 30 to 100 meters depending on the network conditions. The much longer ranges and lower power consumed than other personal area networks (i.e. Bluetooth) make these products suitable for industrial applications in large factory 
settings and in indoor and outdoor installations.

While standard low-bit-rate video encoding is an easy task for today's media processors, it does pose challenges to low-bit-rate ZigBee networks. The most bandwidthefficient media codecs are the most computationally intensive. The nominal rate of the underlying IEEE 802.15.4 standard is $250 \mathrm{kbps}$. Because of the CSMA/CA architecture, the realistic maximum is on the order of half the nominal rate. In practice, the bit-rate could even be lower than the realistic maximum rate.

This research tests video transmission over wireless Zigbee networks. The goal of the research is not to implement a streaming application that requires high datarate. Rather, some video applications such as video surveillance and monitoring will be studied. The size of the network that can be supported over such networks will also be studied. Advanced video encoders, object based segmentation (region-ofinterest) and rate control algorithms are implemented to make the video transmission over IEEE 802.15.4 Zigbee networks feasible. In addition, wireless Zigbee nodes will use the multiple non-overlapping channels that are available in the IEEE 802.15.4 standard. Each node will be equipped with multiple interfaces resulting in increasing the size of the Zigbee network, i.e. icreasing the number of video sources and nodes over the network. 


\subsection{Objective of the Thesis}

This thesis shows how video surveillance over wireless Zigbee networks is possible, by presenting various techniques to solve the problem of low rate and low power introduced in the IEEE 802.15.4 standard. In addition, the thesis aims to provide a way of increasing the size of a video surveillance Zigbee network to accommodate larger areas with an acceptable video quality. The main objectives of the thesis are:

- Evaluating and validating the use of TES traffic modeling (a powerful traffic modeling approach) over wireless Zigbee networks in a video surveillance application:

- Use TES models to replace real low bit-rate video in order to facilitate video transmission over Zigbee networks

- Verify multi-quantization scaling to help in the simulation of rate control video transmission

- Providing a low data loss environment over wireless Zigbee networks by developing a rate control algorithm:

- Develop and validate a rate control algorithm to eliminate the bursty packet losses that occur in variable bit-rate coding

- Then enhance network performance by the addition of a video segmentation (region-of-interest) algorithm

- Make use of the non-overlapping channels in the $2.4 \mathrm{GHz}$ ISM band:

- Extend the use of a multi-channel multi-interface (radio) network solution for IEEE 802.15.4 Zigbee networks 
- Enhance the performance of the multi-channel multi-interface network topology by implementing the Multiple Descriptive Coding (MDC) algorithm and by testing this algorithm over a bursty error channel

All the performance evaluations are done using the ns-2 simulator which consists of $\mathrm{C} / \mathrm{C}++$ and $\mathrm{Tcl}$ coding.

\subsection{Thesis Outline}

The rest of the thesis document is organized as follows:

- Chapter 2 describes some of the technology background concerning wireless Zigbee networks. In addition, the chapter also describes video compression and its different techniques. Finally, it presents some of the work done on multimedia transmission over wireless Zigbee and sensor networks.

- Chapter 3 tests the validity of using TES modeling for low variable bit-rate MPEG4 video traffic over wireless Zigbee networks. After validating the TES methodology over Zigbee networks, the queuing performance of the video model will be compared with the real video.

- Chapter 4 presents a rate control variable bit-rate (RC-VBR) algorithm that combines the benefits of both types of coding (CBR and VBR). The algorithm makes use of the queue to allow rate variability while keeping a small restriction in the bit-rate. In addition, an enhancement of the algorithm is implemented by adding the video segmentation (region-of-interest) technique to increase the number of video sources and therefore increase the topology size of the network.

- Chapter 5 presents a multi-channel multi-radio network topology to increase the size of the wireless Zigbee network and concludes it with the implementation of the Multiple Descriptive Coding (MDC) algorithm. 
- Chapter 6 presents the simulation and performance results of the various algorithms presented in the previous chapters. All simulations are done using the ns-2 simulator which uses $\mathrm{C} / \mathrm{C}++$ and $\mathrm{Tcl}$ for coding.

- Chapter 7 contains conclusions, and suggested future study.

\subsection{Thesis Contributions}

The main contributions of the thesis are as follows:

- TES processes are performed and validated as a suitable modeling approach for low bit-rate MPEG4 video traffic over wireless Zigbee networks. The models are validated for the various types of MPEG4 frames (I, P and B) as well as the overall interleaved video sequence. The thesis then outlines a way to generate video models for the different quantization parameters. By multiplying each frame type by a certain scalar factor which differs according to the video type, the modeling approach does not have to be repeated for every quantization value.

- A rate control variable bit-rate (RC-VBR) algorithm is developed and is shown to outperform traditional encoding (CBR or VBR) techniques over IEEE 802.15.4 networks. The RC-VBR algorithm is based on changing the quantization parameter adaptively according to a feedback loop that measures the size of the buffer and then changes the quantization parameter accordingly.

- A combination of the region-of-interest (ROI) encoding and RC-VBR (i.e. ROIRCVBR) algorithm is presented. The ROI-RCVBR algorithm will enable the Zigbee video network to increase its topology size without losing any significant data. The algorithm is based on segmenting the video objects and sending 
the important parts of the frames that are necessary for a video surveillance application.

- A multi-channel multi-radio network topology is extended to work for wireless Zigbee networks. The technique takes advantage of the multiple free nonoverlapping channels that are available and the low hardware cost in the IEEE 802.15.4 standard. The multi-channel multi-radio network results in a substantial increase in the size of the topology of a wireless Zigbee network.An enhancement of the multi-channel multi-radio network topology is presented. Multiple Descriptive Coding (MDC) is added to the ROI-RCVBR algorithm. Using the enhanced coding technique guarantees the delivery of video content, even in the presence of channel errors which are frequent in real environments. 


\section{Chapter 2}

\section{Overview of Wireless Zigbee Networks and Video Encoding}

This chapter describes some of the technology background concerning wireless Zigbee networks. In addition, the chapter also describes video compression and its different techniques. Finally, it presents some of the work done on multimedia transmission over wireless Zigbee and sensor networks.

\subsection{Wireless Zigbee networks}

\subsubsection{ZigBee Alliance}

The ZigBee Alliance is an association of companies working together to enable reliable, cost effective, and low-power network based on an open global standard. The intended goal of ZigBee Alliance is to create a specific approach on how to build different network topologies with data security features and interoperable application profiles. The Zigbee association is a diverse family of companies providing everything from radio semiconductor chips, to finished products. The number of members in the association is rapidly growing and currently exceeds 100. Among the members, one can find Siemens, Philips, Texas Instruments and Mitsubishi. 
The first specification was approved in 2004 and the first generation of ZigBee products reached the market sometime in 2005 . A big challenge for the alliance is to achieve the interoperability of various products. To solve this problem, the ZigBee Alliance has defined a different set of profiles to categorize their products. For example there is a profile called Home Lighting that exactly defines how different brands of home lighting-products should communicate with each other.

In order to be get access to the specification, one must become a member of the Zigbee Alliance.

\subsubsection{Origins of the name ZigBee}

The name was introduced due to the technique that honey bees use to communicate new-found food sources to other members of the colony. This technique is referred to as the "ZigBee Principle". Using this silent, but powerful communication system, whereby the bees dance in a zig-zag pattern, they are able to share information such as the location, distance, and direction of a newly discovered food source to their fellow colony members.

This principle attracted engineers to use this idea to tackle a complex task by breaking it up into a number of simple tasks.

\subsubsection{Overview of the IEEE 802.15.4 standard}

IEEE 802.15.4 [1] is a standard for low rate wireless personal area networks (LRWPAN) which is designed for ultra-low cost, low power, low complexity and short range communications. This standard offers a maximum nominal rate of $250 \mathrm{~kb} / \mathrm{s}$ and hence can be used for applications that require simple wireless communications such as monitoring, home automation, alarm sensors, health sensors and toys.

IEEE 802.15.4 defines the physical layer and the MAC sublayer of the OSI Zigbee 
stack [1]. It supports devices that consume minimum energy and are designed for low bit-rate applications over a short range of 30 to 100 meters.

\section{IEEE 802.15.4 Device Types}

The IEEE 802.15.4 topology may contain a network coordinator that maintains overall network knowledge. It is the most sophisticated node of the entire 802.15.4 network and requires the most memory and computing power.

Two different devices can participate in a low rate wireless personal area network (LR-WPAN): Full-Function Device (FFD) and Reduced-Function Device (RFD).

\section{- Full-Function Device (FFD)}

The FFD is capable to operate as as a PAN coordinator, and it can talk to RFDs or other FFDs.

\section{- Reduced-Function Device (RFD)}

The RFD is intended for very simple applications and cannot operate as a PAN coordinator. Moreover, the RFD can only communicate with FFDs in the system. Thus, two RFDs cannot exchange information with each other

\section{Network topologies of IEEE 802.15.4 devices}

IEEE 802.15.4 devices may operate in either one of two topologies: the star or the peer-to-peer topology as illustrated in figure 2.1. These two network topologies can be mixed to produce a Zigbee mesh topology.

\section{- Star Topology}

In a star topology, communication can only occur between a certain device (RFD or FFD) in the system and the PAN coordinator. Applications such 

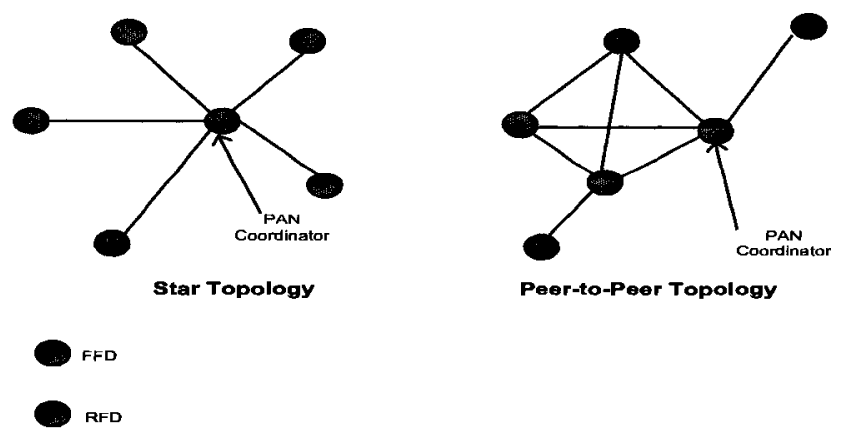

Figure 2.1: Star and peer-to-peer topology examples

as personal computer peripherals and wireless body area networks (WBANs) would typically employ the star topology.

\section{- Peer-to-Peer Topology}

Peer-to-peer topologies allow devices to communicate with each other without contacting or going through the PAN coordinator. It also allows more complex formations like the cluster-tree and mesh networking topologies. Industrial and environmental monitoring are examples of applications that use the peer-to-peer topology.

\section{Zigbee OSI stack overview}

The Open System Interconnection (OSI) reference model was developed by the International Organization for Standardization (ISO) as a model for the computer protocol architecture, and as a framework for developing protocol standards. The model is used to separate networking into several distinct functions that operate at different layers in order to simplify the standard. Each layer is responsible for performing a specific task or set of tasks, and dealing with the layers above and below it. However, the 
IEEE 802.15.4 standard only defines the Physical (PHY) and MAC layers. The upper layers of networking and application have been left for the application developers. An 802.15.4 LR-WPAN device comprises a Physical layer, which contains the radio frequency (RF) transceiver along with its low-level control mechanism, and a MAC sub-layer that provides access to the physical channel for all types of transfer.

An illustration of the general OSI-model and where ZigBee is defined in the model can be seen in Figure 2.2 .

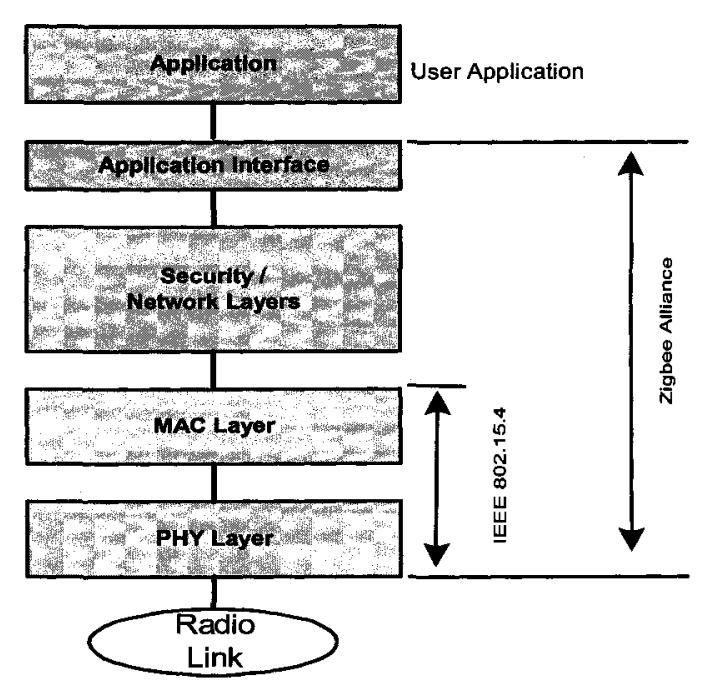

Figure 2.2: Zigbee OSI Layers

As mentioned earlier, the IEEE 802.15.4 standard only defines the Physical (PHY) and MAC layers.

- Physical layer

The IEEE 802.15.4 standard operates in three frequency bands each supporting different data rates and different numbers of channels. They can be described as follows:

- $868 \mathrm{MHz}$ band which supports a data rate of $20 \mathrm{kbps}$ and contains 1 channel. 
- $916 \mathrm{MHz}$ band which supports a data rate of $40 \mathrm{kbps}$ and contains 10 channels.

- $2450 \mathrm{MHz}(2.4 \mathrm{GHz})$ band which supports a data rate of $250 \mathrm{kbps}$ and contains 16 channels.

The features of the PHY layer are activation and deactivation of the radio transceiver, energy detection (ED), link quality indication (LQI), channel selection, clear channel assessment (CCA), and transmitting as well as receiving packets across the physical medium.

Since the $2450 \mathrm{MHz}$ frequency band supports the highest data rate and the largest number of channels, it will be the chosen band for the video transmission. The offset quadrature phase shift keying (OQPSK) is used as the modulation technique. Transmission distances are about $30 \mathrm{~m}$ for non-line of sight and $80 \mathrm{~m}$ for line of sight which is suitable for video surveillance over a personal network.

- Mac sublayer

IEEE 802.15.4 is based on CSMA (channel sense multiple access) with two modes of operation. The unslotted-CSMA (beaconless mode) where nodes are considered peers and any node can send a packet if the channel is available, and the slotted-CSMA (beacon enabled mode) which contains a super frame structure with beacons for time synchronization.

The MAC layer is responsible for beacon management, channel access, guaranteed time slot (GTS) management for high priority communications, frame validation, acknowledged frame delivery, association, and disassociation.

Acknowledgments are optional in IEEE 802.15.4. They are used to confirm the reception of packets. Retransmission is done if no acknowledgments are received by the sender. After a certain number of retransmissions, the sender will drop the packet. 
Zigbee offers best effort traffic, which means no priority levels or quality of service (QOS) is provided. This makes video transmission more challenging.

- Security Layer

There are three types of security modes defined: unsecured mode, access control list mode and secured mode.

- Unsecured mode: No security used.

- Access control list mode: No encryption used, but the network rejects frames from unknown devices.

- Secured mode: In the secured mode the devices can use the following security services:

* Access control list.

* Data encryption using the Advanced Encryption Standard (AES) 128 bit encryption algorithm.

* Frame integrity using Message Integrity Code (MIC) to protect data from being modified by parties without the cryptographic key.

* Sequential freshness using ordered sequence of inputs to reject frames that have been replayed. When a frame is received, the freshness value is compared with the last known freshness value.

- Zigbee Network Layer

Zigbee devices may operate in either one of two topologies: the star or the peerto-peer topology. ZigBee can use mesh networking, which may extend over a large area and contain thousands of nodes. Mesh networking is a combination of star and peer-to-peer network topologies. The routing protocol optimizes the shortest and most reliable path through the network and can dynamically 
change, so as to take evolving conditions into account. This enables an extremely reliable network, since the network can heal itself if some nodes are disabled.

\section{- Application Layer}

Zigbee is designed for applications that require low cost, low power and low datarate. Applications for such networks include agricultural, residential, remote diagnostics, home automation, personal healthcare, security and surveillance applications.

\subsubsection{IEEE 802.15.4 Interference}

The IEEE 802.15.4 standard uses the 2.4GHz Industrial Scientific and Medical (ISM) unlicensed band to provide global availability. This ISM band is commonly used for other standards such as IEEE 802.11b/g (WLAN) and IEEE 802.15 .1 (Bluetooth). Therefore, unrestricted access to the ISM band exposes the IEEE 802.15.4 devices to a high level of interference. Since the IEEE 802.15.4 and the IEEE 802.11 were introduced for different purposes, they can coexist within each others communication and interference ranges. For instance, the IEEE 802.15.4 network is mostly used for low rate applications such as control and surveillance networks while the IEEE 802.11 network is used for applications that require high data rates such as wireless audio/video (A/V) streaming. Therefore, the coexistence of the IEEE 802.15.4 and the IEEE 802.11 networks needs to be assessed and a solution needs to be introduced.

Some previous work studied the coexistence of the IEEE 802.15.4 and WLAN using real measurements and simulations. In [39], it is shown that IEEE 802.15.4 has little or no effect on IEEE 802.11 networks whereas IEEE 802.11 has serious impact on IEEE 802.15.4 if no attention is taken [40]. In [41], the impact of interference of WPAN over both WPAN and WLAN are studied using OPNET simulations. It was 
shown that the interference will result in high data losses as a result of the coexistence.

\section{Operation in the ISM bands}

The IEEE 802.15.4 defines three physical layers; the $2.4 \mathrm{GHz}, 868 \mathrm{MHz}$ and 915 $\mathrm{MHz}$ frequency bands. The unlicensed Industrial Scientific Medical (ISM) $2.4 \mathrm{GHz}$ band is available worldwide, while the $868 \mathrm{MHz}$ and $915 \mathrm{MHz}$ bands are available in Europe and North America respectively. A total of 27 channels with three different data rates are defined for the IEEE 802.15.4: 16 channels with a data rate of 250 kbps at the $2.4 \mathrm{GHz}$ band, 10 channels with a data rate of $40 \mathrm{kbps}$ at the $915 \mathrm{MHz}$ band, and 1 channel with a data rate of $20 \mathrm{kbps}$ at the $868 \mathrm{MHz}$ band. Different data rates offer better choices for different applications in terms of energy and cost efficiency. For example, while $250 \mathrm{~kb} / \mathrm{s}$ is required for some computer peripherals and interactive toys, a lower data rate like $20 \mathrm{~kb} / \mathrm{s}$ can meet the requirements of many other envisioned applications such as various sensors, smart tags, and consumer electronics. The relationship between the IEEE 802.11b (non-overlapping sets) and the IEEE 802.15.4 channels at the $2.4 \mathrm{GHz}$ is illustrated in Figure 2.3.

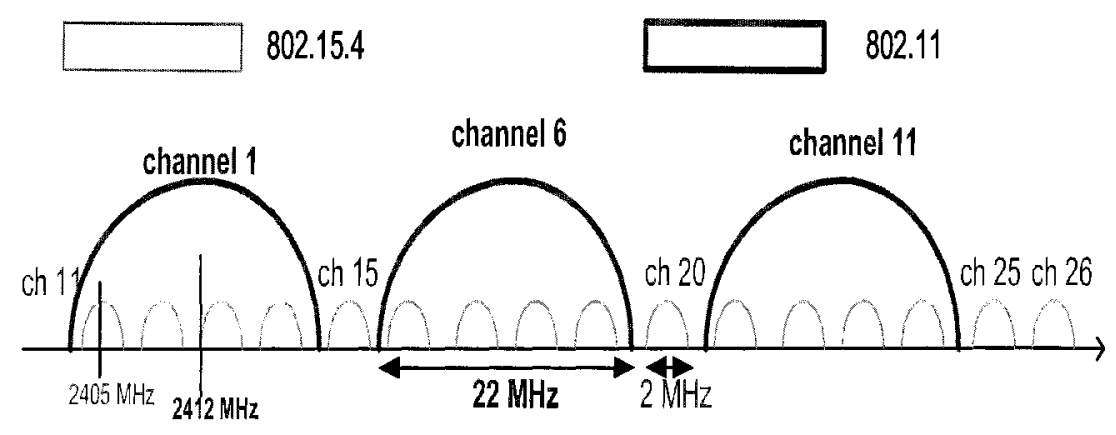

Figure 2.3: LR-WPAN and WLAN interferece

Figure 2.3 shows the operation frequency spectrum of both IEEE 802.11 and IEEE 802.15.4 networks in the 2.4 GHz ISM band. The IEEE 802.11 standard has 11 
channels each of which occupies $22 \mathrm{MHz}$. Up to 3 channels can be used simultaneously without mutual interference. As illustrated in the figure, channels 1,6 and 11 can be used by the IEEE 802.11 devices to eliminate the mutual interference. On the other hand, the IEEE 802.15.4 standard defines 16 channels (2 MHz), channels 11 through 27 , in the $2.4 \mathrm{GHz}$ ISM band all of which can be used simultaneously without mutual interference. The IEEE 802.15.4 standard recommends using the channels that fall in the guard bands between two of the three adjacent non-overlapping IEEE 802.11 channels or above these channels to prevent interference between the IEEE 802.15.4 and the IEEE 802.11. From the figure, it is shown that 4 of the 16 channels will have the minimal interference which in most cases will be enough to cover a wide region unless more IEEE 802.15.4 networks are added.

In general two or more heterogeneous systems will coexist in a single networking environment. This is because different communication systems can be used for different applications. Especially, the IEEE 802.11 (WLANs) will most probably coexist with IEEE 802.15.4 (WPANs) in the same Industrial Scientific Medical (ISM) band. Interference will have more effect if the LR-WPANs communication range is expanded using the ad-hoc mode (peer-to-peer clustering). The interference results in substantial data losses especially in IEEE 802.15.4 networks because it's more susceptible to interference. As shown in figure 2.3, this problem can be solved by choosing frequency channels that do not overlap with the IEEE 802.11 channels. Moreover, multiple IEEE 802.15.4 channels can be used to increase the network throughput and eliminate the interference caused by other IEEE 802.15.4 networks. 


\subsection{Overview of video encoding standards}

In the coding process, each picture is divided into blocks, groups of blocks and macroblocks. Each macroblock is encoded with respect to a specific quantization parameter $\mathrm{Q}$. The quantization parameter determines the spatial resolution of the encoded picture, i.e. by increasing the $Q$ value, the bit rate and image quality will decrease. There are two coding methods used in general: Constant bit-rate (CBR) and Variable bit-rate (VBR) coding. In CBR coding, the video is transmitted at a constant rate with bounded delay. In VBR coding, the video is transmitted with rate variations.

\subsubsection{Constant bit-rate (CBR) coding}

This method depends on changing the quantization parameter at the Group-ofPicture (GOP) or frame level which is done by fixing the quantity of bits allocated to each GOP or frame [28]. Figure 2.4 shows an example of CBR coding for a monitoring video sequence. The figure shows how the quantization parameter $\mathrm{Q}$ changes from one frame to another for the same video sequence. The number of packets allocated for every frame varies since some of the scenes contain more activity than others. The video sequence used does not vary widely because no scenes change during the sequence. Therefore, the $\mathrm{Q}$ values do not vary widely as would be scene in a video sequence with different scene changes, especially for big buffers. However, for

small buffers, (50 Zigbee packets as in figure 2.4), as the Q value keeps on changing frequently, the image quality keeps on changing quite often. This is one of the major drawbacks for the CBR coding method, where the video quality changes every time more bits are needed. In addition, one of the drawbacks of CBR is the delay (200ms for a reasonable value) introduced by the smoothing buffer that has the role of eliminating the rate variability. This buffer is used for guaranteeing picture quality. Thus, there is a tradeoff between variability in quality and delay introduced by the 
smoothing buffer.

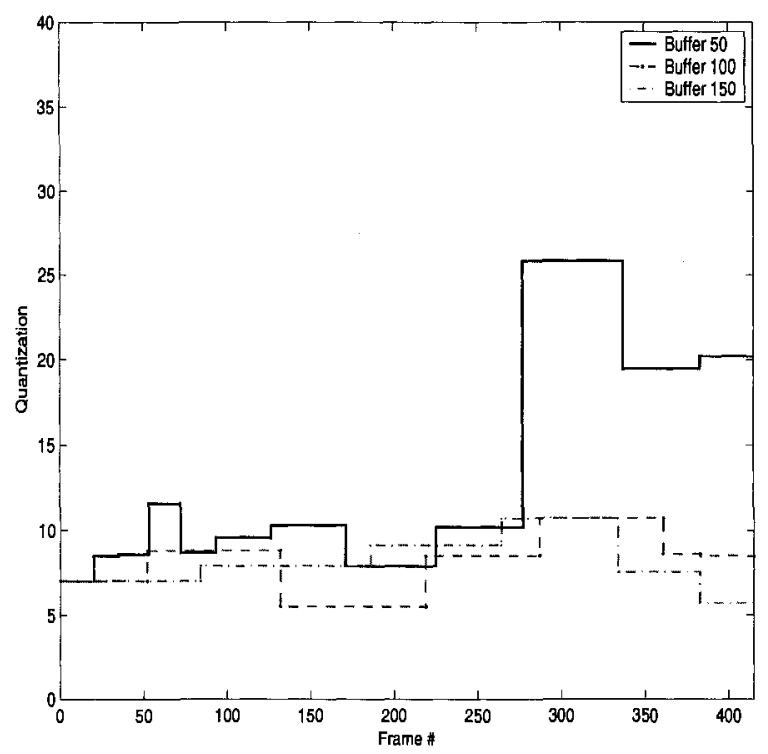

Figure 2.4: Frame Index vs. Quantization Parameter

\subsubsection{Variable bit-rate (VBR) coding}

Variable bit rate (VBR) coding is one of the coding techniques that require high bandwidth across the network. This gives rise to autocorrelated traffic streams of bursty nature. Previous work showed that autocorrelated traffic has worse impacts on queuing systems than uncorrelated traffic [3] [4].

VBR coding was introduced due to the spread of packet switching, where avoiding the rate variability is not necessarily a problem. Therefore, VBR coding can be used where the image quality will not change during the whole video sequence, i.e. the $\mathrm{Q}$ value of the sequence remains constant over the same video sequence. The rate variability depends on the activity and complexity of the scenes. The quantization parameter has an effect on the frame length and hence on the quality of the video. If the quality increases ( $Q$ value decreases), the number of bytes representing the frames increase. 
Since Zigbee networks have low data rates, rate variability could cause high data losses. Moreover, if the number of hops or number of video sources changes during the video transmission, frames may get lost. For instance, the quality of the video decreases if the number of hops or the number of video sources increases. Even if the number of hops or video sources decreases, the quality can be increased by decreasing the $Q$ value. Therefore, there should be a method that combines the benefits of both CBR and VBR coding.

\subsubsection{Overview of the MPEG4 video encoding standard}

The Motion Picture Experts Group (MPEG) started the MPEG4 project in 1993 with the goal of producing low-bit rate streams, though the standard has since been expanded to include a broader range of bit-rates and applications. The most notable addition to MPEG4 since MPEG2 is the capability to represent video sequences as a composition of independent audio-visual objects [25]. However both standards use similar techniques to exploit spatial and temporal redundancy.

MPEG4 is the latest standard for inter-frame compression and storage of digital video from the MPEG group. It is expected to be the predominant encoding for wireless and internet video. It offers significant advantages over current formats due to its ability to encode video sequences on an object-by-object basis at a wide range of bit-rates. Furthermore, MPEG4 has some error-resilience capabilities, such as resynchronization markers, data hiding and reversible VLC that are useful for the transport of a video stream in an error-prone network.

Figure 2.5 shows a block diagram of an MPEG4 encoder. It permits compression of rectangular sized image sequences at varying levels of input formats, frame rates and bit rates. While spatial redundancy is exploited via transform coding, temporal redundancy is exploited via block based motion estimation and compensation techniques. 


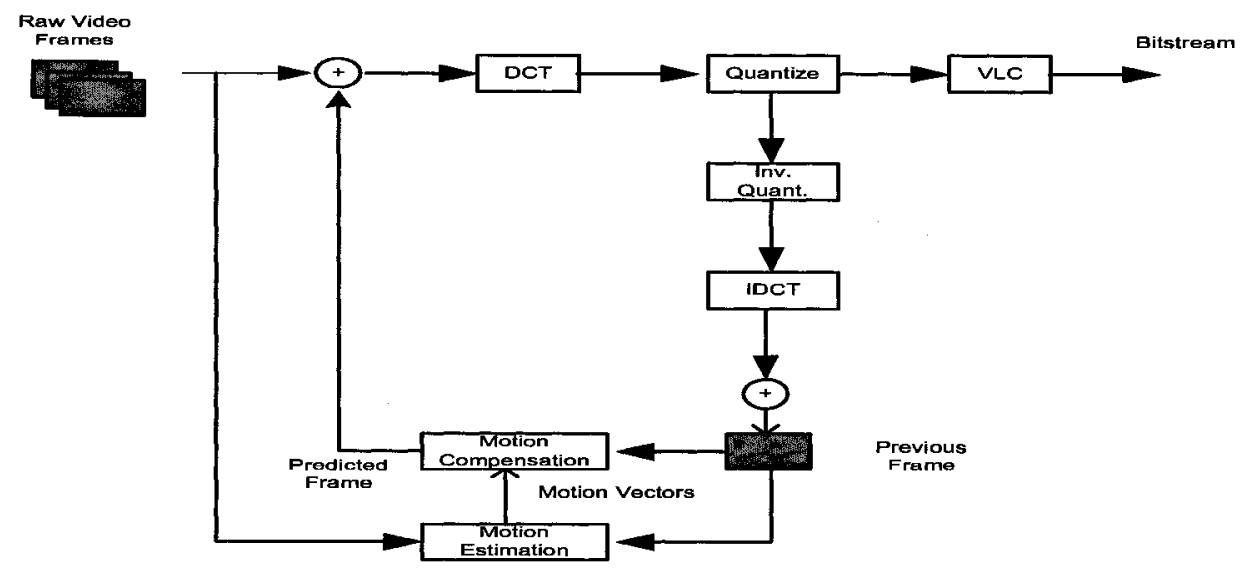

Figure 2.5: MPEG4 Overview

Figure 2.6 shows the dependencies that exist within an MPEG4 bitstream. "IVOPs" or "I-frames" are intra-coded frames, coded independently from other frames, so they act as reference frames and do not exploit temporal redundancy. "P-VOPs" or "P-frames" are coded predictively from the closest previous reference I or P frame, and "B-BVOPs" or "B-frames" are coded bi-directionally from the preceding and succeeding reference frame.

Frames in MPEG4 are arranged in a Group of Pictures (GOP). A GOP consists of one I-frame, some P-frames that are related to it and optionally some B-frames between the I and P frames. Therefore, if an I-frame is lost or corrupted, the whole corresponding GOP will be of no use even if all other $\mathrm{P}$ and $\mathrm{B}$ frames are received correctly. Whereas, if P-frames are lost, this will only affect previous or successive B-frames. If B-frames are lost this will not affect any other frames and the overall performance of the video will still be fine

Dependent frames are coded by performing motion estimation, which includes a 


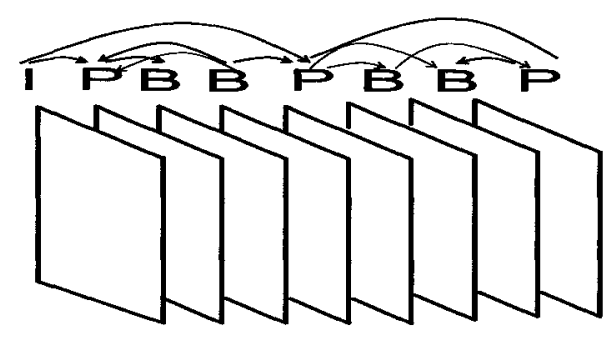

Figure 2.6: MPEG4 frame sequences.

process called block matching and motion compensation. Block matching is the process by which $16 \times 16$ pixel blocks of the dependent image, called macroblocks, are matched against macroblocks in the reference frame to find the closest possible match for that block. The amount by which each block has moved in relation to the reference frame is encoded in a motion vector. The combination of the macroblocks from the reference frame and motion vectors produce a rough estimate of the temporally dependent frame, which is how motion compensation is done. The difference between this estimate and the actual frame is encoded as a motion residual, along with the motion vectors. At this point, the motion vector search range should be considered. Since the motion vector search range represents the macroblock dimensions, the computational energy increases due to the increase in search area. The typical optimal search range varies between sequences depending on the motion intensity factor. It is seen that if the search range becomes large, the coding quality decreases and the source rate increases. This can be attributed to the fact that as the search range increases, large motion vectors are being selected, and more bits are used to code the motion-vector differences. 


\subsection{Multimedia applications over wireless Zigbee and sensor networks}

Wireless sensor networks (WSNs) have drawn the attention of the research community in the last few years, driven by a wealth of theoretical and practical challenges. This growing interest can be largely attributed to new applications enabled by largescale networks of small devices capable of collecting data from the surrounding environment, performing simple processing on the collected data and transmitting it to remote locations. Significant results in this area of research have lead to a surge of civil and military applications. Nowadays, most deployed wireless sensor networks measure scalar physical phenomena like temperature, pressure, humidity, or the location of objects. In general, most of the applications have low bandwidth demands, and are usually delay tolerant.

The availability of low-cost hardware cameras and microphones has advanced the development of Wireless Multimedia Sensor Networks (WMSNs), i.e. networks of wirelessly interconnected devices that are able to ubiquitously retrieve multimedia content such as video and audio streams, still images, and scalar sensor data from the environment. Although sensor networks cannot be used for high quality video transmission, they can be used in surveillance and monitoring applications that require minimum video quality.

With the rapid improvements in software and hardware, the sensor nodes can be equipped with audio or video data collection modules. Moreover, to be able to form WMSNs, sensor nodes should also be able to store, process, and fuse multimedia information from various sensor sources.

Several architectures for wireless image sensor networks have been introduced in the past. In 2005, Cao et al. [11] proposed an image sensor architecture, in which an FPGA connects to a VGA (640480 pixel) CMOS imager to carry out image acquisition 
and compression. An ARM7 microcontroller processes images further and communicates to neighboring sensors via an ultra-low-power and data rate transceiver. The Cyclops image capturing module [12], was designed for extremely light-weight imaging and can be interfaced with a host mote such as Crossbow's MICAz motes [13]. Cyclops was developed as an add-on CIF (320240 pixel) CMOS camera module board, which hosts an on-board 8- bit microcontroller and 64 Kbytes of static and 512 Kbytes of flash memory for pixel-level processing and storage. In [14], smart camera mote architecture is presented. The sensor uses an FPGA as its central processing unit, a VGA CMOS imager, and 10 Mbytes of static and 64 Mbytes of flash memory to perform early-vision, i.e. pixel-level, tasks.

A mote architecture with minimal component count was introduced in [15] at Stanford's Wireless Sensor Networks Lab. It deploys an ARM7 microcontroller at its core, up to 2 Mbytes of flash memory, and a $2.4 \mathrm{GHz}$ IEEE 802.15 .4 radio. Unlike the motes mentioned above, this mote pioneers the concept of multiple vision sensors as it can host up to four low-resolution (3030 pixel) image sensors and two CIF CMOS camera modules. Both types of vision sensors feature a serial interface thus eliminating the need for additional FPGA or CPLD devices.

In [16], a hardware design for distributed smart camera motes is implemented for a surveillance application based on the IEEE 802.15.4 Zigbee architecture. A smart camera mote architecture was designed for in-node processing is introduced. The aim was to facilitate distributed intelligent surveillance. The mote architecture targets the provision of sufficient processing power and an adequate vision system while minimizing component count and power consumption. Low power consumption is an important design objective to enable mobile surveillance applications using batterypowered camera motes.

Wireless multimedia sensor and Zigbee networks will open new interesting applications which will not be limited to home automation and monitoring, rather they 
will be used for applications such as multimedia surveillance sensor networks, traffic avoidance, enforcement and control, automated assistance for the elderly and family monitors and person locator services. 


\section{Chapter 3}

\section{Introduction to Video Traffic Modeling using Transform-Expand-Sample (TES)}

\section{Processes}

This chapter provides an overview of TES processes and tests the validity of using TES modeling for low variable bit-rate MPEG4 video traffic over wireless Zigbee networks. After validating the TES methodology over Zigbee networks, the queuing performance of the video model will be compared with the real video.

Most common approaches employed for video source modeling are based on fluid models and autoregressive models. In the fluid approach, a video stream is modeled as having a fixed rate, modulated by some stochastic fluid process [6]. This method is complex and requires a lot of knowledge in video modeling since it involves large number of states, parameters and transition rates. In the autoregressive approach, a video bit rate is modeled as a weighted some of a finite number of previous bit rates. Frater et al. [7] employs the DAR (Discrete Autoregressive) scheme to model full-motion movies, including "Star Wars". The DAR model was also applied in [8] to a variety of conference video records and was shown to work well. One potential drawback of 
autoregressive models is that while they aim to fit the empirical autocorrelation function, they lack a systematic way of fitting the empirical marginal distribution. Since traffic burstiness can be caused by the shape of the marginal bit rate distribution as well as that of the empirical autocorrelation function, it is important to capture both first-order and second-order properties of the empirical data. TES processes are transformed autoregressive processes which are used to model video bit rates at the frame level and the group-of-block level [5].

The model used in the research depends on the TES modeling methodology to accurately capture both first-order and second-order statistics of the empirical bit rate record; more specifically, the model approximates well the marginal distribution and the autocorrelation function of the bit rate process, simultaneously, and model sample paths (frame sizes) show a strong "resemblance" to the empirical record. In addition, TES modeling is systematic and intuitive, i.e. simple and does not require a lot of knowledge about video modeling. In addition, a big advantage of TES processes is that autocorrelation functions can be calculated using a mathematical formula which makes the modeling phase faster. TEStool [9] is a graphical user interface that allows modeling stationary sequences using the TES methodology.

In [17], an investigation of TES modeling and multiplexing of MPEG traffic over ATM networks was performed. In [18] [19] H.261 video compression is modeled using the TES methodology. In [20], TES modeling is used to generate models for high bit-rate MPEG4 traffic. However, TES modeling is still not validated for low bit-rate video traffic. 


\subsection{Overview of Transform-Expand-Sample (TES) processes}

TES processes [22] [23] are autoregressive modulo-1 schemes which are easy to implement on a computer with low computational complexity. The TES methodology is versatile, which means that it can generate data that matches the stationary distribution and capture the effect of correlations of any set of given observations of a time series.

For any real number $x$, let $\langle x\rangle=x-\lfloor x\rfloor$ be the fractional part of $\mathrm{x}$, where $\lfloor x\rfloor=\{$ integern $: n \leq x\}$. which is called the integral part of $x$. The derivation of TES models is done in two phases. The first phase considers forming correlated sequences (background sequences) which are uniformly distributed in $[0,1)$ as follows:

$$
\begin{gathered}
U_{n}^{+}= \begin{cases}U_{0} & \text { if } x=0 \\
<U_{n-1}^{+}+V_{n}> & \text { if } n>0\end{cases} \\
U_{n}^{-}= \begin{cases}U_{n}^{+} & \text {if n even } \\
1-U_{n}^{+} & \text {if } \mathrm{n} \text { odd }\end{cases}
\end{gathered}
$$

where $U_{0}$ is a uniform random variable in $[0,1)$ and $\left\{V_{n}\right\}$ is a sequence of independent and identically distributed random variables called the innovation sequences with probability density function $f_{v}$. $U_{0}$ is independent of each element of $\left\{V_{n}\right\}$.

TES processes come in two forms: $\left\{T E S^{+}\right\}$and $\left\{T E S^{-}\right\} .\left\{T E S^{+}\right\}$representing the sequence $U_{n}^{+}$covering the range $[0,1)$ and $\left\{T E S^{-}\right\}$representing the sequence $U_{n}^{-}$ covering the range $[-1,0)$. Both can be computed recursively as shown above.

The second phase is to generate the foreground sequence which is done by some transformation called the distortion D. 


$$
X_{n}^{+}=D\left(U_{n}^{+}\right), X_{n}^{-}=D\left(U_{n}^{-}\right)
$$

Since $U_{n}$ has a uniform distribution on $[0,1)$, the transformation $D$ allows us to generate a sequence that resembles the real sample using the inversion technique represented by $D=F^{-1}$. The sequences $\left\{X_{n}^{+}\right\}$and $\left\{X_{n}^{-}\right\}$are both correlated and identically distributed. Therefore, an empirical histogram is used to represent the marginal distribution of empirical data.

The innovation sequence $\left\{V_{n}\right\}$ has two representations. The basic representation is taken as:

$$
V_{n}=L+(R-L) Z_{n},-0.5 \leq L<R \leq 0.5
$$

where $Z_{n}$ is an iid sequence uniformly distributed on $[0,1)$ and hence $V_{n}$ is uniformly distributed on $[\mathrm{L}, \mathrm{R})$. Another representation of the innovation sequence can be done using $(\alpha, \phi)$ parameters as follows:

$$
\alpha=R-L, \phi=\frac{R+L}{R-L}
$$

where $0<\alpha \leq 1$ and $0 \leq|\phi| \leq \frac{1}{\alpha}-1$

The basic method for calculating the density function $f_{v}$ of the innovation sequence $\left\{V_{n}\right\}$ is as follows:

$$
f_{v}(x)=\sum_{k=1}^{K} 1_{\left[L_{k}, R_{k}\right)}(x) \frac{P_{k}}{R_{k}-L_{k}},
$$

where $1_{A}$ is the indicator function of set $\mathrm{A}, \mathrm{K}$ is the number of histogram cells, $\left[L_{k}, R_{k}\right)$ is the support of cell $\mathrm{k}$ with width $R_{k}-L_{k}$ and $P_{k}$ is the probability estimator of cell $\mathrm{k}$. This means that each $V_{n}$ is a mixture of uniform random variates on $\left[L_{k}, R_{k}\right)$ with probability $P_{k}$. 
TES modeling is done by first capturing the autocorrelation of the input time series using innovation density function $f_{v}$ and then applying a smoothing technique using the stitching transform $(\zeta)$.

The last step results in a distorted background sequence which is then inverted to a foreground sequence using the inverse of the histogram distribution which always guarantees the matching of the marginals. The task of finding an appropriate innovation density that approximates the empirical autocorrelation is carried out via a heuristic search using TEStool [9].

\subsection{TES modeling of MPEG4 video}

The MPEG4 [25] standard uses both spatial (intra-frame) and temporal (inter-frame) redundancy encoding because of the similarities of the successive frames for the same scene. Frames that are encoded using spatial redundancy are the frames that contain the most important part of the scene. The frames are reduced using Discrete Cosine Transformation (DCT), quantization and then entropy coding. In temporal redundancy, only the differences between successive frames are encoded using frame differencing, motion estimation and motion-compensated prediction [25].

Compressed frames can either be I-frames which are intra-coded frames, P-frames which are predicted frames and B-frames which are bidirectional frames. I-frames contain the most information which is why they are encoded independently. P-frames that depend on previous I or P frames, are less important than I-frames but more important than B-frames. B-frames are the least important frames and they depend on previous and successive I or P frames. Frames in MPEG4 are arranged in Groups of Pictures (GOP). A GOP consists of one I-frame, some P-frames that are related to it and optionally some B-frames between the I and P frames. 
In this section, an MPEG4 traffic generation model, using TES processes is developed. MPEG4 traces are generated by encoding an indoor surveillance video at a rate of 10 frames per second (fps) with different quantization parameters and a GOP of 12 frames between two consecutive I-frames.

The traffic model of the real video shown in this section is done with a quantization parameter of 7 (any other value could have been chosen and would have produced similar results). The quantization parameter determines the spatial resolution of the encoded picture. By increasing the $\mathrm{Q}$ value, the bit rate and image quality decrease.

The steps for generating the traffic model with a quantization parameter of 7 are explained in this section. Figures 3.1, 3.2 and 3.3 show the empirical (real) MPEG4 video trace statistics of the frame sizes. Figure 3.1 shows the sample path (frame size) statistics of the sequence in bits. Figure 3.2 shows the empirical histogram of the sequence. Figure 3.3 shows the empirical autocorrelation function of the sequence. Since the overall video sequence is not stationary as can be seen from the previously mentioned figures, and because of the periodic nature of the autocorrelation function, a $T E S^{+}$model is applied for the I and B frame statistics and a $T E S^{-}$model is applied for the $\mathrm{P}$ frame statistics as it was performed in [18].

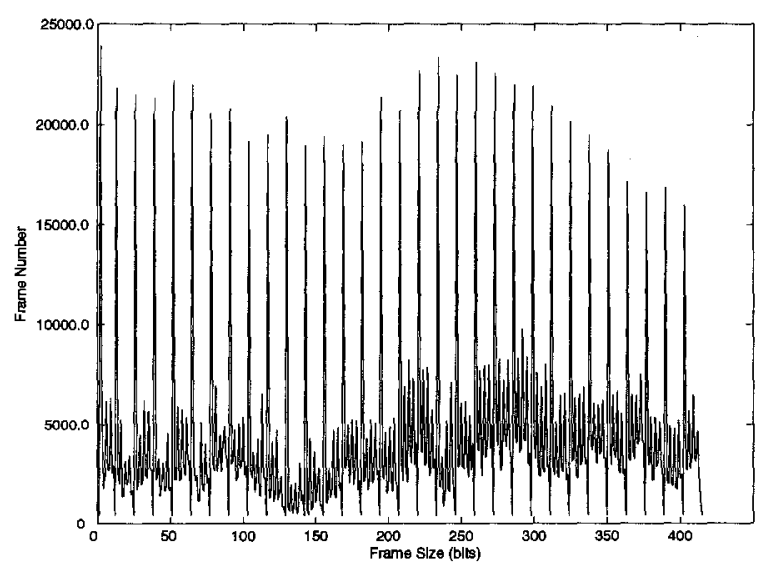

Figure 3.1: Empirical video frame trace with $\mathrm{Q}=7$

Figures 3.4, 3.5 and 3.6 show respectively the TES model of the sample path (frame 


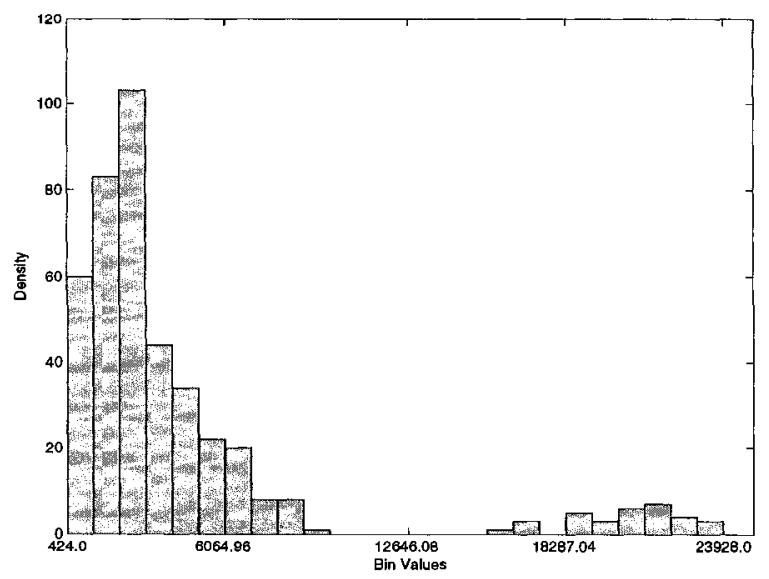

Figure 3.2: Empirical histogram with $Q=7$

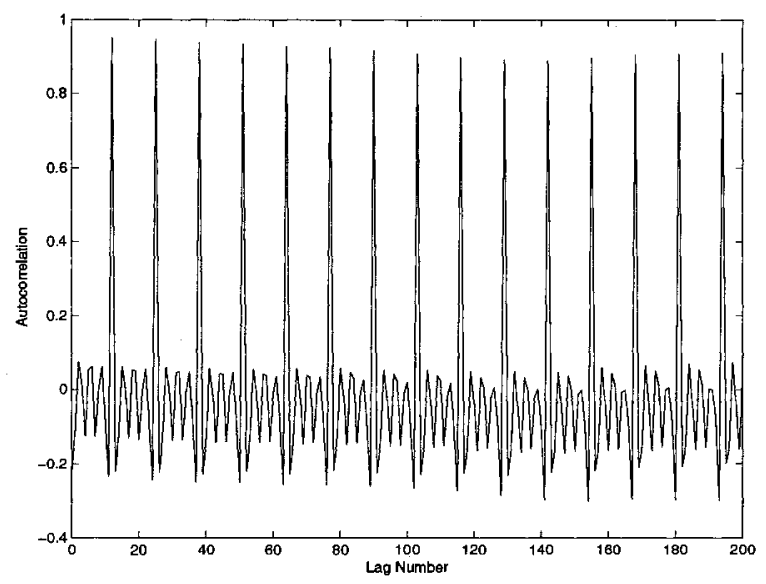

Figure 3.3: Empirical autocorrelation function with $Q=7$

sizes), histogram and autocorrelation function of the I-frames. Figure 3.7 shows an appropriate innovation density function $f_{v}$ that approximates the autocorrelation function of the I-frames as much as possible. An appropriate innovation density function is carried out via a heuristic search using TEStool [9].

From figures 3.4, 3.5 and 3.6, it can be shown that the empirical and modeled histograms always match while there are small differences in the autocorrelation function and sample path. The same steps were taken to generate the P-frame and B-frame traffic models.

After generating the individual frame sizes from the TES models, the overall 


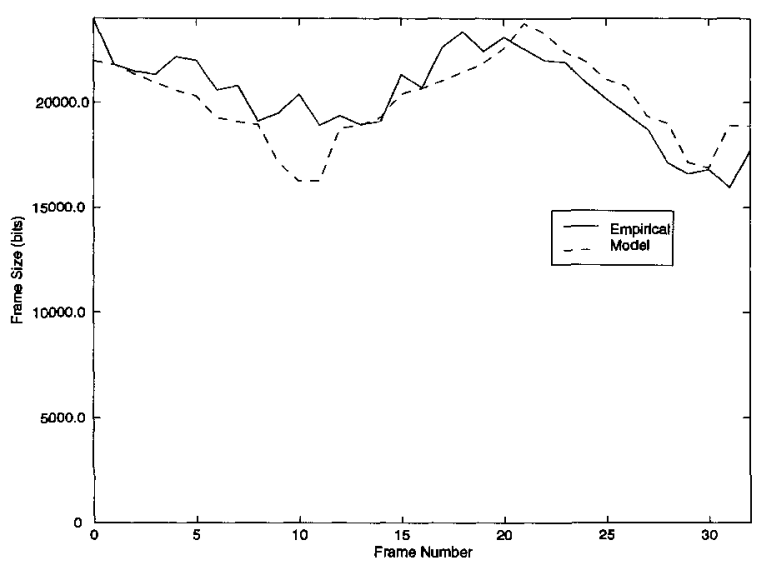

Figure 3.4: Original and generated model of I-frames

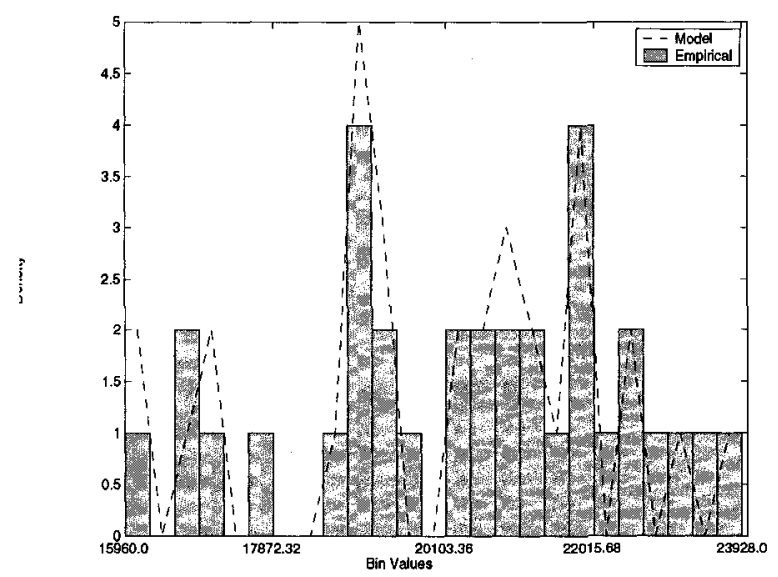

Figure 3.5: Original and generated model of the I-frames histogram

modeled MPEG4 video trace is generated. This is done by interleaving the sample paths of the video frames by following the original video sequence.

Table 3.1 shows the mean of the original and modeled I, P, B and overall sequences as well as the parameters used for modeling.

The results of the testool for the I, P, B and overall real and modeled samples are shown in figures $3.8,3.9,3.10$ and 3.11 respectively. Every figure is divided into four windows: window w1 of the figure shows the sample path of the frame sizes, window w2 shows the autocorrelation function, window w3 shows the histogram of the distribution of the frame sizes and window w4 shows an appropriate innovation 


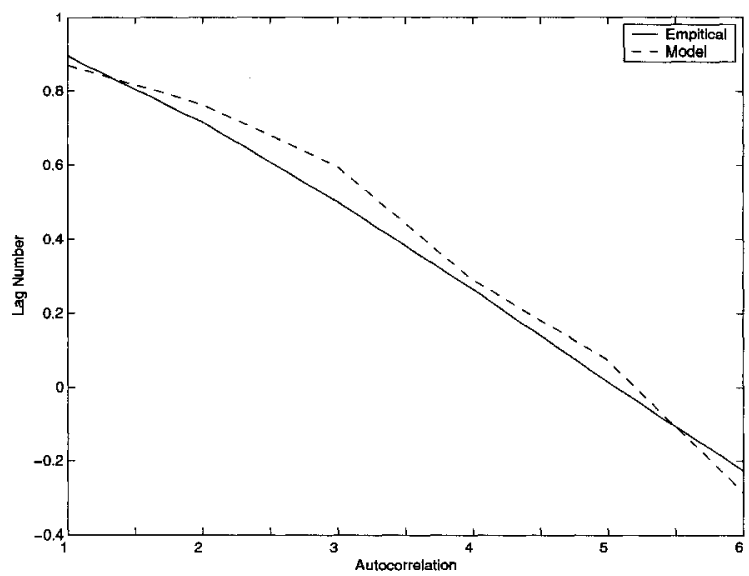

Figure 3.6: Original and generated model of the I-frames autocorrelation function

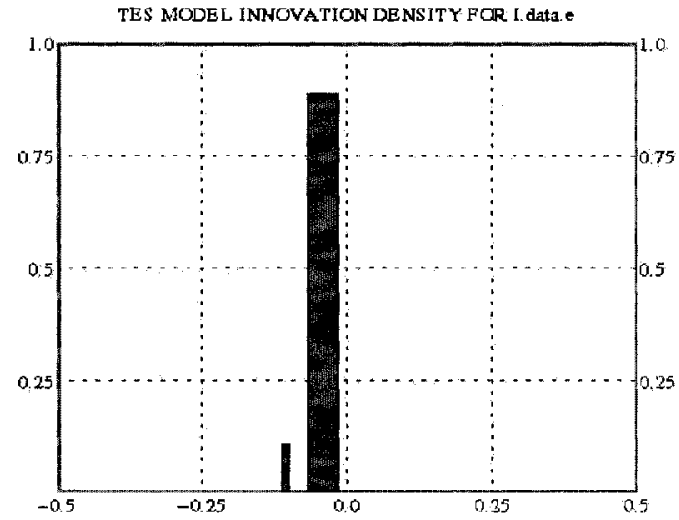

Figure 3.7: Innovation function of the I-frames model

function that generates an approximate model. From the figures, we observe that the histogram always matches the original data while there is little difference in both the autocorrelation function and sample path. 


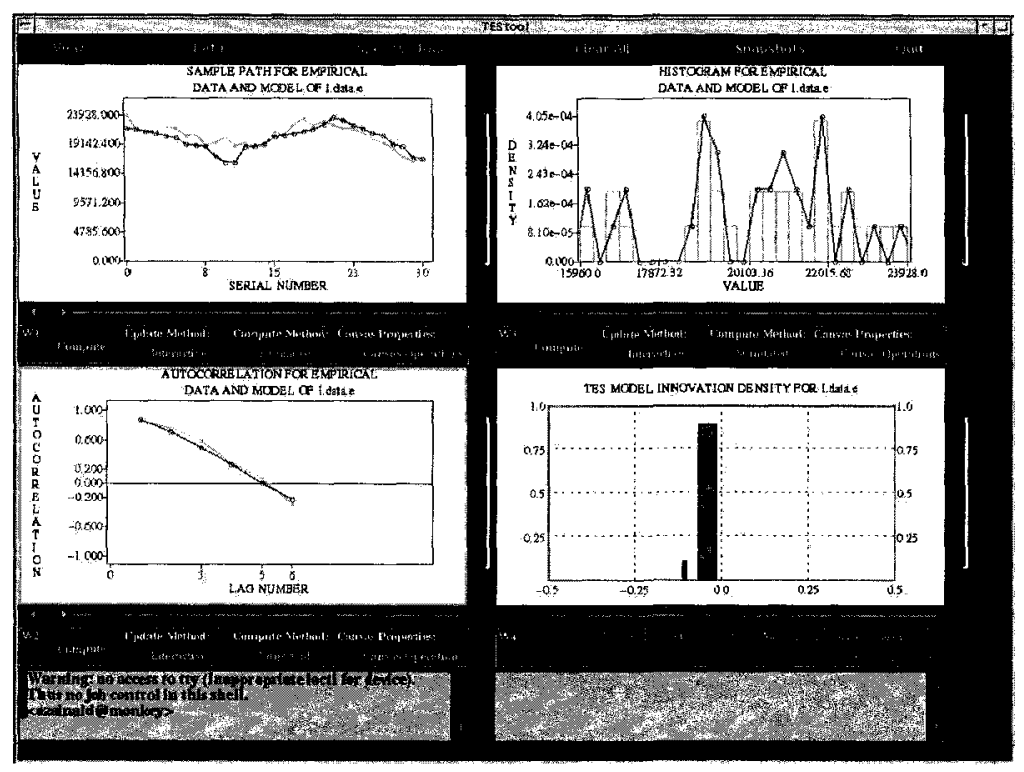

Figure 3.8: Modeled vs. Real TES data for the I frames as appears in the testool

\subsubsection{Verification of TES modeling scaling factor of MPEG4 video frames}

Similar modeling results occurred when using different quantization parameters. By comparing the different quantization parameters, the autocorrelation function of the real and modeled video, match closely. Whereas, the frame sizes of the real and modeled video differ. However, the shapes of the traces are similar with just a scaling factor difference. Hence, in order to generate a traffic model with a certain quantization parameter, it is not required to do so for every quantization value. An example

Table 3.1: Statistics and parameters of the models

\begin{tabular}{|l|l|l|l|l|}
\hline \multirow{2}{*}{} & \multicolumn{2}{|l|}{ Mean (bits) } & \multicolumn{2}{l|}{ Parameters } \\
\cline { 2 - 5 } & Original & Model & $U_{0}$ & $\zeta$ \\
\hline \hline I Frames & 20490 & 20146 & 0.460 & 0.60 \\
P Frames & 5573 & 5701 & 0.810 & 0.60 \\
B Frames & 2512 & 2556 & 0.590 & 0.40 \\
Overall(interleaved) & 4442 & 4499 & & \\
\hline
\end{tabular}




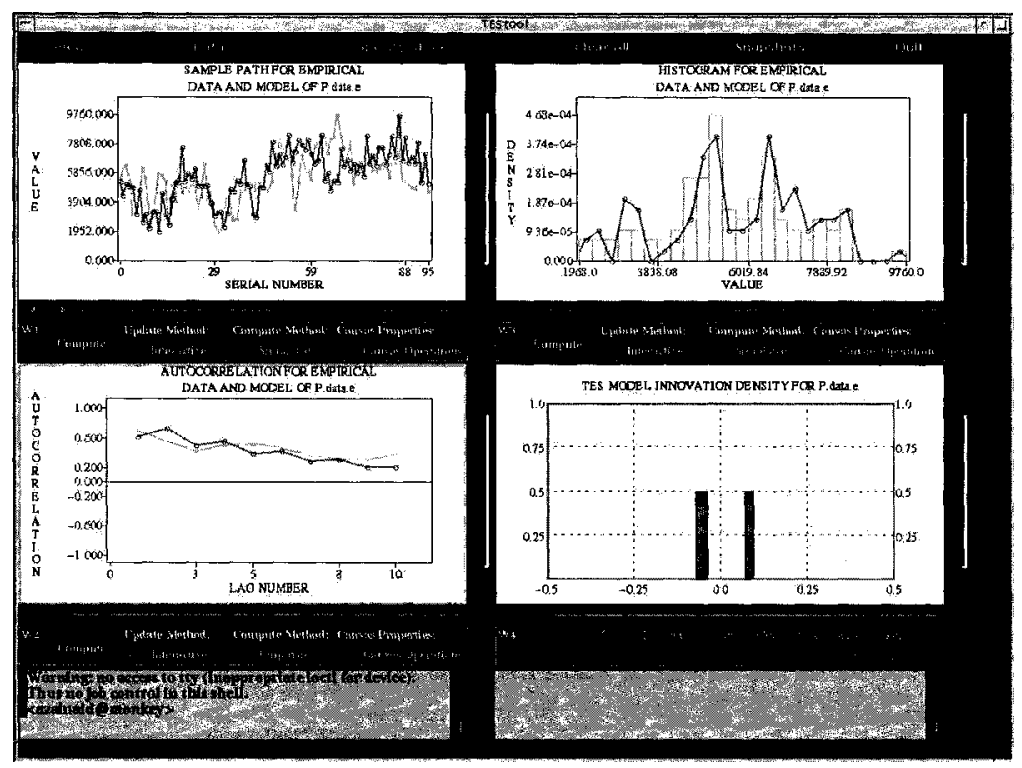

Figure 3.9: Modeled vs. Real TES data for the $P$ frames as appears in the testool

is shown in figure 3.12 which is taken from the testool to compare the video sequence statistics for quantization parameters 7 and 12 .

In other words, only one model of the video is required, while the rest can just be multiplied by a scaling factor that depends on the video type. This scaling factor is different for the I, P and B frames. Figure 3.13 illustrates the relationship between the bit rate per frame versus the quantization parameter, with the knowledge that the bit rate is normalized to the $Q=7$ case. 


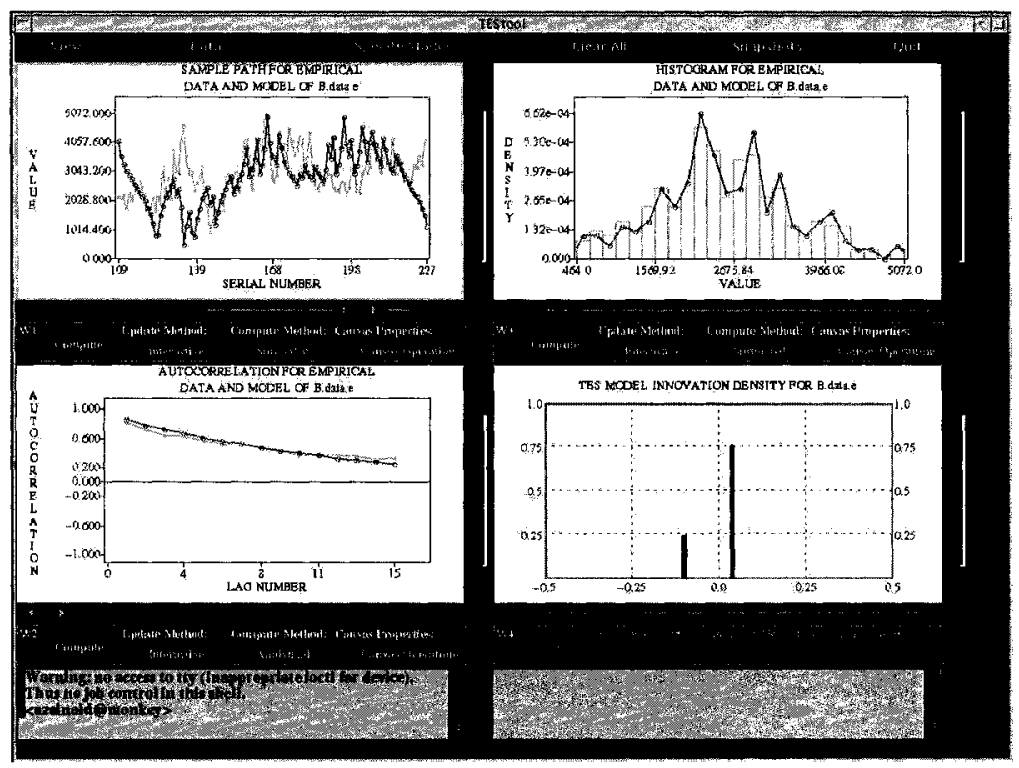

Figure 3.10: Modeled vs. Real TES data for the B frames as appears in the testool

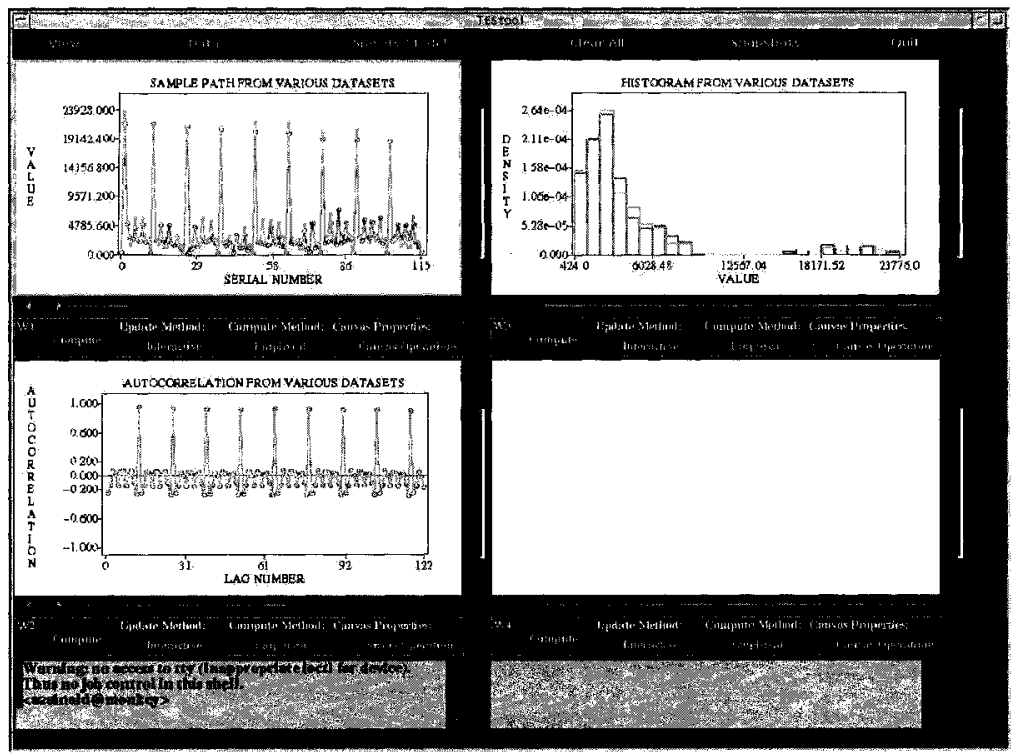

Figure 3.11: Modeled vs. Real TES data for the overall frame sequence as appears in the testool 


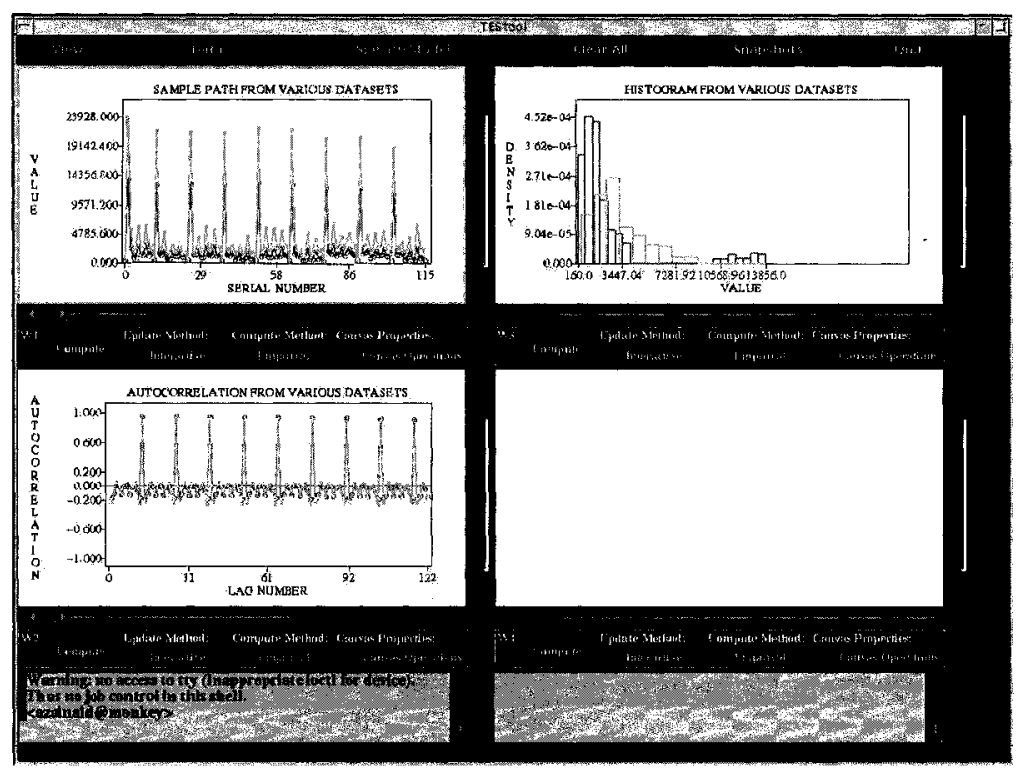

Figure 3.12: Frame sequence statistics as appears in the testool for $\mathrm{Q} 7$ and 12

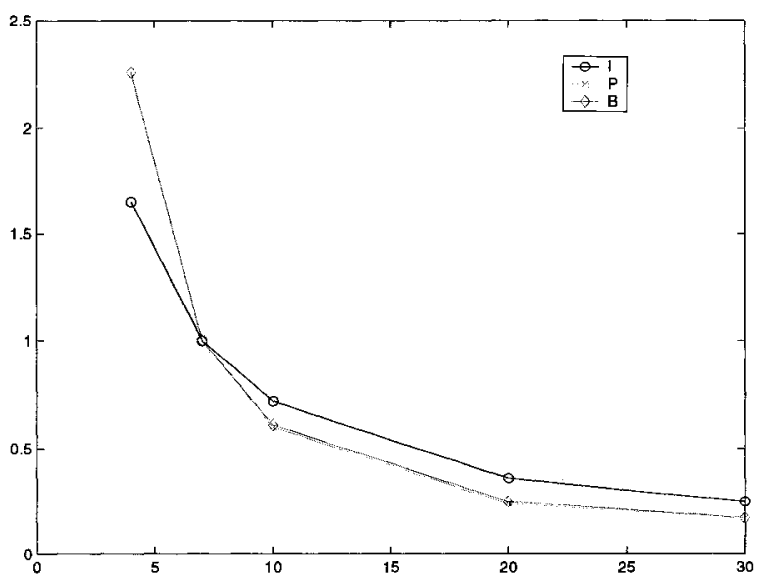

Figure 3.13: Scaling Factor vs. Quantization parameter 


\section{Chapter 4}

\section{Rate Control Variable bit-rate (RC-VBR) over Wireless Zigbee Networks}

Constant bit-rate (CBR) coding restricts the output bit-rate and tries to maintain this rate throughout the video transmission. This results in a continuous change in the video quality. Whereas, variable bit-rate (VBR) coding preserves the quality of the video by keeping the value of the quantization parameter unchanged. However, VBR encoding results in a high traffic burstiness due to a big difference between the intra-frame and inter-frame sizes.

This chapter presents a rate control variable bit-rate (RC-VBR) algorithm that combines the benefits of both types of coding (CBR and VBR). The algorithm makes use of the queue to allow rate variability while keeping a small restriction in the bit-rate. In addition, an enhancement of the algorithm is implemented by adding the video segmentation (region-of-interest) technique to increase the number of video sources and therefore increase the size of the network. 


\subsection{Rate Control Video Transmission}

Some studies considered adaptive forward error correction to calculate the rate needed to encode the VBR video. In [29], an analytical rate control model using adaptive forward error correction (FEC) schemes is proposed for wireless networks. A similar approach is proposed in [30] by considering mobile ad hoc networks. These schemes introduce redundancy, depending on the current channel conditions. Accordingly, the throughput available at the application layer changes. It increases as channel conditions improve, and decreases as channel conditions worsen. In [30],the encoding parameters were tuned to adapt the video source transmission rate to the available throughput. However, the previous schemes are not suitable for wireless Zigbee networks since they introduce a large amount of overhead due to the FEC packets added during poor channel conditions.

Other studies considered computing a relationship between the quantization parameter, data size and video complexity. In [31], a generic relationship to compute the quantization parameter from the video frame sizes and I-frame spatial complexity is proposed. This relationship provides a mechanism to select the quantization value for an I-frame (with different spatial complexity) before actually encoding it. Experiments show that the adaptive intra-frame quantization algorithm reduces the amount of frame skipping significantly with a comparable average peak signal-to-noise ratio (PSNR), thus improving the overall subjective quality of the reconstructed video. However, this method requires the quantization parameter to be continuously computed, resulting in high power consumption that is not suitable for Zigbee networks.

There were also studies that considered Automatic Repeat Request (ARQ) to design their rate control algorithms. In [32] [33], a source rate control algorithm is implemented according to the buffer fullness at the transmitter side. Channel feedback is considered in order to enhance the rate control algorithm by changing 
the rate produced by the encoder according to the channel conditions. The previous studies are similar to the approach used by the developed rate control. However, no feedback is needed from the destination even if the channel conditions become worse. The reason being that the buffer fullness will be known. This approach is more appropriate than the previous work for wireless Zigbee networks because no additional packets are needed for feedback. Moreover, the computational power is minimal in agreement with the low power requirement of the IEEE 802.15.4 standard.

\subsection{Rate control variable bit-rate (RC-VBR) algo- rithm over wireless Zigbee networks}

The rate control VBR algorithm developed combines the benefits of both types of coding by using the queue to allow rate variability but at the same time keep a small restriction in the bit-rate.

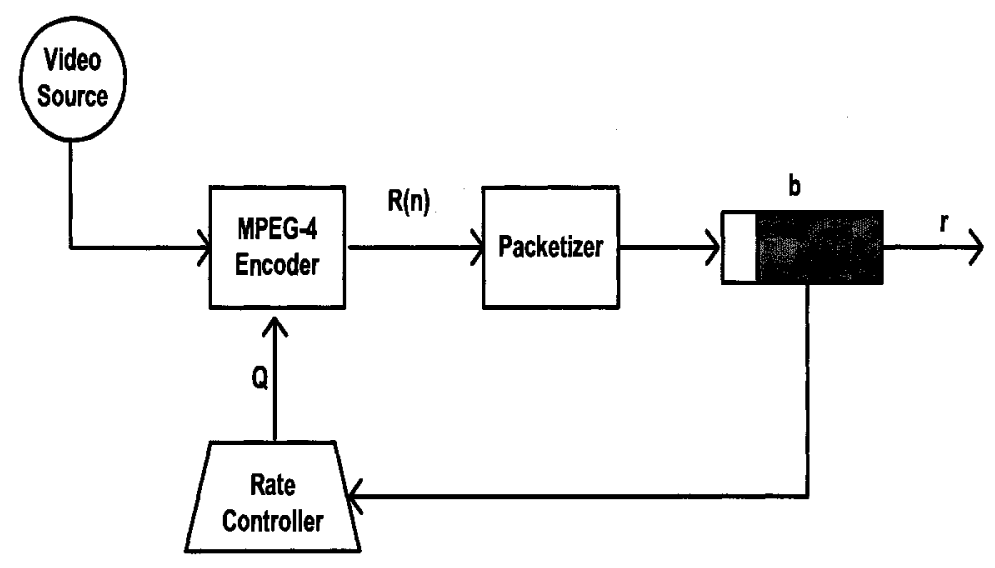

Figure 4.1: RC-VBR encoder

Figure 4.1 shows the complete MPEG4 encoder when rate control VBR is used. No additional buffer is added to the encoder, resulting in an extra delay, that is known to occur in CBR coding. The rate controller is used to make sure that the 
buffer remains at a certain range by continuously checking the size of the queue. The output rate of the buffer is $\mathbf{r}$ (in packets per second) and the buffer size is $b$ (in packets). The rate controller will keep a counter $\mathrm{X}$ representing the number of packets in the buffer. The counter increases or decreases to certain defined thresholds. The rate controller informs the encoder if these thresholds are reached so as to change the encoding parameters. The quantization parameter Q changes on a per GOP level. However, the rate change of $\mathrm{Q}$ is much less in RC-VBR coding than in CBR coding because of the introduction of the buffer size counter and the thresholds. This results in a consistent video quality with the addition of a rate controller.

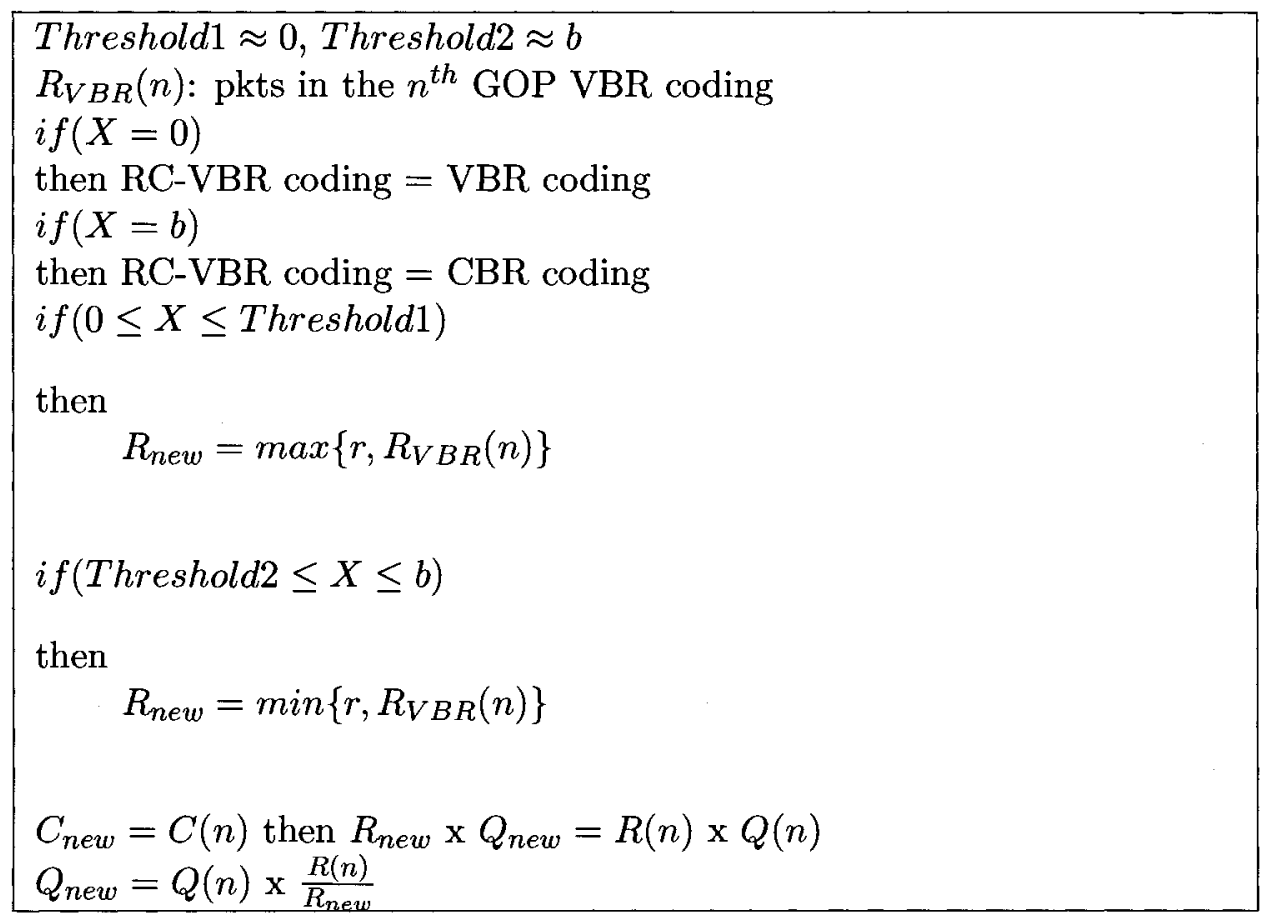

Figure 4.2: RC-VBR algorithm

The RC-VBR functionality is similar to the one found in [34]. The number of packets in the buffer is kept as a counter $\mathrm{X}$ to make sure that the upper and lower thresholds are met $(0 \leq X \leq b)$. $\mathrm{R}(\mathrm{n})$ is the number of packets generated, while $\mathrm{Q}(\mathrm{n})$ is the quantization parameter for the n-th GOP. 
The RC-VBR algorithm makes sure that $\mathrm{X}$ is never close to 0 or $\mathrm{b}$, by monitoring the packet size counter $\mathrm{X}$ and comparing it with a threshold. Therefore, if $\mathrm{X}$ is not close to 0 or $\mathrm{b}$, the quality of the video remains unchanged. Whereas, if the $\mathrm{X}$ counter crosses the thresholds (i.e. becomes close to either 0 or b), the RC-VBR algorithm changes the video quality value by either increasing or decreasing it respectively.

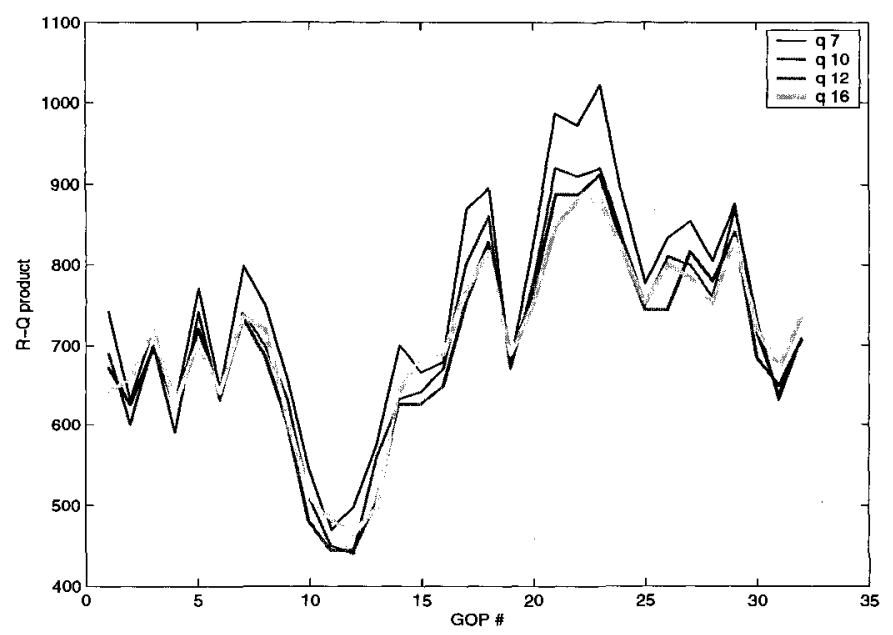

Figure 4.3: GOP Index vs. R-Q Product

The $\mathrm{Q}$ value will change for different reasons. One reason is the change in scene complexity, i.e an object appearing or disappearing in surveillance networks. Another reason could be a change in the number of hops or a change in the channel conditions. In the aforementioned cases, the quantization parameter values should be changed to make sure that the object can be tracked. A good measure for the scene complexity is the R-Q product [34].

Figure 4.3 shows the R-Q product versus the GOP number for different quantization parameters. From the figure, it is shown that the R-Q product of each GOP is almost the same for various $Q$ values. The product difference between the various GOPs is due to the difference in frame complexity. However, the R-Q product for every GOP is a constant which represents the complexity of the scene. Let: 
$C(n)=R(n) \cdot Q(n)$ and from figure 4.3 , it can be seen that $\mathrm{C}(\mathrm{n})$ is practically independent from $\mathrm{Q}$. The full RC-VBR algorithm is summarized in figure 4.2.

\subsection{Region-of-interest based rate control variable bit-rate (ROI RC-VBR) algorithm over wire- less Zigbee networks}

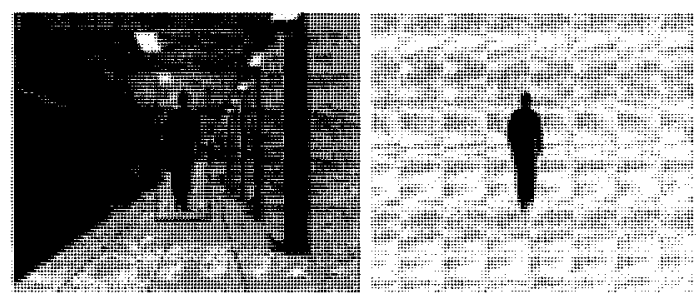

Figure 4.4: a) Real video frame sample b) Video frame using video segmentation

In a conventional surveillance network, the static background regions in the video frames are encoded and transmitted over the network even if they do not contribute significant information. Video surveillance sequences contain long periods of inactivity and short periods of activity limited to a small region of the frame. Therefore, a method for object segmentation will reduce the video traffic over the network. Thus, this method is appropriate for low bit-rate networks such as Zigbee networks. The method can be produced by analyzing and encoding important regions of the frames and hence transmitting smaller frame parts, resulting in an increase of the topology size (i.e. number of video camera sources).

In [35], region-of-interest coding is used for JPEG2000 image transmissions over surveillance networks. In [37], a spatial-temporal segmentation technique was introduced to encode an MPEG-2 content based encoded video. The segmentation 
technique used in this paper is similar to the one in [37]. However, the segmented object (i.e. region-of-interest) frame is passed to the rate control VBR (RC-VBR) encoder instead of the MPEG-2 encoder to make sure the video output rate remains within certain boundaries. This algorithm is called the region-of-interest rate control VBR (ROI-RCVBR) algorithm. Figure 4.4a shows the original frame while figure $4.4 \mathrm{~b}$ shows a segmented frame where only the person object is encoded. 


\section{Chapter 5}

\section{Multi-channel Multi-radio over Wireless Zigbee Networks}

Various studies were performed to solve the problem of interference by using the multiple channels available in IEEE 802.15.4 networks. In [42], Won et al. proposed an adaptive interference avoidance scheme by forming a mesh network over multiple IEEE 802.15.4 devices. In [43], Pollin et al. proposed an adaptive multi-channel solution in case of interference with other IEEE 802.11 networks by considering a random channel discovery. This approach makes it difficult for other devices to change their frequency channels to the new one. In [44], the problem of interference was solved by making every cluster group use the same channel and in case of interference, the whole group changes their channel by using the same pseudo random number generator.

This chapter presents a multi-channel multi-radio network topology to increase the size of the wireless Zigbee network and concludes it with the implementation of the Multiple Descriptive Coding (MDC) algorithm. 


\subsection{Related work of multi-channel multi-radio net- work topology}

Wired networks still overcome wireless networks when it comes to sustaining bandwidth. Wireless network technologies with multiple channels and multiple interfaces are now being focused on because they allow high performance improvement with a relative low cost and complexity. Current multi-channel standard technologies such as IEEE 802.11 and IEEE 802.15.4 are mostly being used in single channel mode. We expect that if a multi-channel multi-interface system is implemented, it will lead to a

dramatic increase in throughput. For example, IEEE 802.11 has two standards, the a and b/g which allow up to 12 and 3 non-over lapping orthogonal frequency channels respectively. The non-overlapping channels can be used simultaneously. Similarly, the IEEE 802.15.4 allows up to 16 non-over lapping channels to be used simultaneously.

In order to use this technology, additional radio hardware is required, but this will not affect the cost since large scale production of hardware products is cheap. Each interface is assigned with an available channel. In our work, we will use up to two interfaces with a multiple channel option. A further increase of the number of interfaces and channels could increase the throughput (per user). However, the complexity and cost of the implementation will increase.

In previous work, proposals have been made to modify the MAC layer to support multiple channel networks [45] [46]. Such networks avoid interference and allow parallel transmission. Other studies of multi-radio networks that have been proposed, suggest the use of multiple interfaces within nodes in a network by assigning identical channels to all nodes [49]. Another set of studies suggested the use of multiple radios with directional antennas in which the MAC was modified to support beam forming or separate radios to communicate within the network [50]. Such studies are not practical since the standard is modified. 
Different architectural design implementation for multi-channel multi-radio technology were implemented. In the Hyacinth project, Raniwala et al. [51] [52] proposed routing and interface assignment algorithms for wireless mesh networks. Mesh nodes use multiple radio channels simultaneously by equipping nodes with multiple NICs that work directly with the interface and use software modifications to manage packet routing and interface channel assignment. The channel assignment consisted of centralized and distributed assignment techniques. In [52], it was shown that by implementing two interfaces and assigning each interface with a single channel, the throughput increases by a factor of 2. Raniwala furthermore suggested the use of channel assignment for multi-radio and multi-channel mesh networks. It was shown that by using only 2 NIC cards per node and around 5 channels for the network, the throughput increased by a factor of 6 to 7 compared to single channel architecture. The hyacinth project was implemented both in ns-2 and in a real testbed.

Other work were also implemented in ns-2 for multi-channel multi-interface networks. The MITF project was carried out at Rio de Janeiro University. The goal of the project was to implement an AODV protocol over a multi-radio ad hoc network using ns-2.28. The TENS [53] project was done at the Indian institute of Technology. The project considered ns-2.19b to implement multiple interfaces over IEEE 802.11 while using multiplexing.

All previous work were designed for IEEE 802.11 since it is the most known ad hoc standard. However, there was a need for a flexible, modular, well integrated and easily configurable design. Therefore, a recent study [54] was developed to allow a large variety of technologies, protocols and roles (sensors, WLAN, WiMax) to be mixed together. The MW-Node [54] project was also designed for IEEE 802.11 networks. However, it was easy to integrate it with the IEEE 802.15.4 network so as to work with multiple channels and multiple interfaces. 
There are three interface assignment strategies when implementing interface assignments. They are static, dynamic and hybrid strategies.

The static strategy assigns the channel to interface permanently, or for long intervals according to the switching time. Within this strategy, there exist two sub-classes, which are common channel approach and varying channel approach [49] [51]. In the common channel approach, interfaces of all nodes are assigned to a common set of channels, while in the varying channel approach, interfaces may be assigned to a different set of channels.

The dynamic assignment strategy allows any interface to be assigned to any channel, and interfaces can frequently switch from one channel to another [46] [47]. A coordination mechanism is required to ensure that two nodes are on the same channel when they communicate with each other. Examples of coordination mechanisms are a node visiting a common channel periodically or the use of pseudo-random sequences. An advantage of this strategy is the ability to switch an interface to any channel while having the potential to cover many channels with few interfaces.

The hybrid assignment combines static and dynamic channel assignment strategies by applying static assignment to some interfaces and dynamic to others [48].

\subsection{Extension for multi-channel multi-radio over IEEE 802.15.4/Zigbee networks}

Currently, there is no work for implementing multi-channels and multi-interfaces over IEEE 802.15.4 Zigbee networks. Therefore, a multi-channel multi-interface wireless Zigbee network is implemented in this chapter and is tested in the next chapter using a simulation study. All video cameras will implement the ROI-RCVBR algorithm which was studied in section 4.3 since it will produce a low bit-rate video which is 
suitable for Zigbee networks.

\subsection{Multiple Description Coding (MDC) over a multi-channel multi-interface Zigbee networks}

Since 16 channels are in the ISM band in the IEEE 802.15.4 standard, the functionality of the Zigbee video nodes can be enhanced by adding one or more interfaces to them. Therefore, video transmission can happen in multiple streams.

Some studies proposed to send the entire video over one path and keep the other path as a backup. Other studies proposed that the entire video can be transmitted over both paths so that if something happens to the main path, the sink (receiver) can alternate to the other path. However, these approaches are not suitable for wireless Zigbee networks since by sending the entire video over a single path, which is the first approach, the energy will be dissipated from the nodes that are part of the main path. Whereas in the second approach, the network will be flooded by video data since the entire video is transmitted in both directions. Therefore, two main concepts were introduced: multiple layer coding and multiple descriptive coding.

Multiple Layer Coding (MLC) and Multiple Descriptive Coding (MDC) are two efficient coding approaches to multi-path transmission [55] [56]. The two approaches provide better error resilience than single layer coding over multi-path transmission. For single layer coding, packet losses may cause error propagation that sharply degrades the overall quality of the reconstructed video. 


\subsubsection{Multiple Layer Coding (MLC)}

In Multiple Layer Coding (MLC), the video stream is divided into a base layer ( $\mathrm{I}$ frames) and enhancement layer (P and B frames) [56]. Different protection mechanisms can be adopted for the base layer and enhancement layer. In MLC, the base layer (I frames) is essential. Therefore, forward error coding (FEC) or automatic repeat request (ARQ) is used to support the base layer. This is because the base layer is required to regenerate the original video. On the other hand, less restriction can be applied to the enhancement layer since it is not as important as the base layer in recovering the transmitted video.

\subsubsection{Multiple Descriptive Coding (MDC)}

In Multiple Descriptive Coding (MDC), the video source stream is split into multiple entities called descriptions, where each of the entities is decodable in a stand-alone fashion [55]. As more of these descriptions are received, the more the transmitted video can be restored. This means that the different descriptions of MDC are complementary, i.e. packet losses in one description can be recovered from other descriptions as long as the same parts of the multiple descriptions are not lost simultaneously.

\subsubsection{Related Studies of multiple coding mechanisms}

There are some research studies comparing MDC and MLC. R. Singh et al. [57] presents a comparative study of MDC and MLC in a wide range of scenarios with different network conditions. Using simulations, it was shown that MDC outperforms MLC over a wide range. Y.Wang [58] compares the performance of MDC and MLC over general wireless networks. Simulation results demonstrate that using a combination of Layer Coding and ARQ gives a better performance than MDC. However, 
MDC results in a better quality than MLC without ARQ. J. Pan [60] presents a comparative study of MDC and MLC. Using ns-2 simulations, it was shown that MDC is more suitable for symmetrical links while MLC is better in links with different channel conditions.

\section{Implementation of MDC over multi-channel multi-radio Zigbee networks}

The assumption of this thesis is that errors occur randomly in wireless channels. Therefore, MLC coding is not chosen since if the error occurs over the channel that is responsible for the base layer (I-frames) path transmission, the overall video will not be reconstructed during channel errors. Whereas, by using MDC, the overall video can be reconstructed because if errors occurred in one of the channel paths, the description received from the other path can be recovered in a stand-alone fashion. In addition, it is better to use MLC coding with forward error correction (FEC). This is not suitable for wireless Zigbee networks because FEC requires high data rates. Hence, MDC is used over a multi-channel multi-interface Zigbee video transmission. The combination of multiple path transport and MDC can provide robustness against severe network conditions and enable load balance.

Figure 5.1 shows the complete node architecture used in the simulations. The upper portion of the figure is responsible for the region-of-interest rate control VBR (ROI-RCVBR) algorithm studied in section 4.3. This portion is only present in the Zigbee surveillance camera sensors while the lower portion is present in all Zigbee sensor nodes including the aggregation and sink nodes. Multiple Descriptive Coding (MDC) is added to the ROI-RCVBR algorithm for the sake of completeness. The lower portion of the figure shows the Zigbee protocol stack used in the node. An important observation is that every output descriptor from the MDC will pass to a separate transceiver (interface). Each transceiver operates on a different frequency channel to form the multi-channel multi-interface topology. 


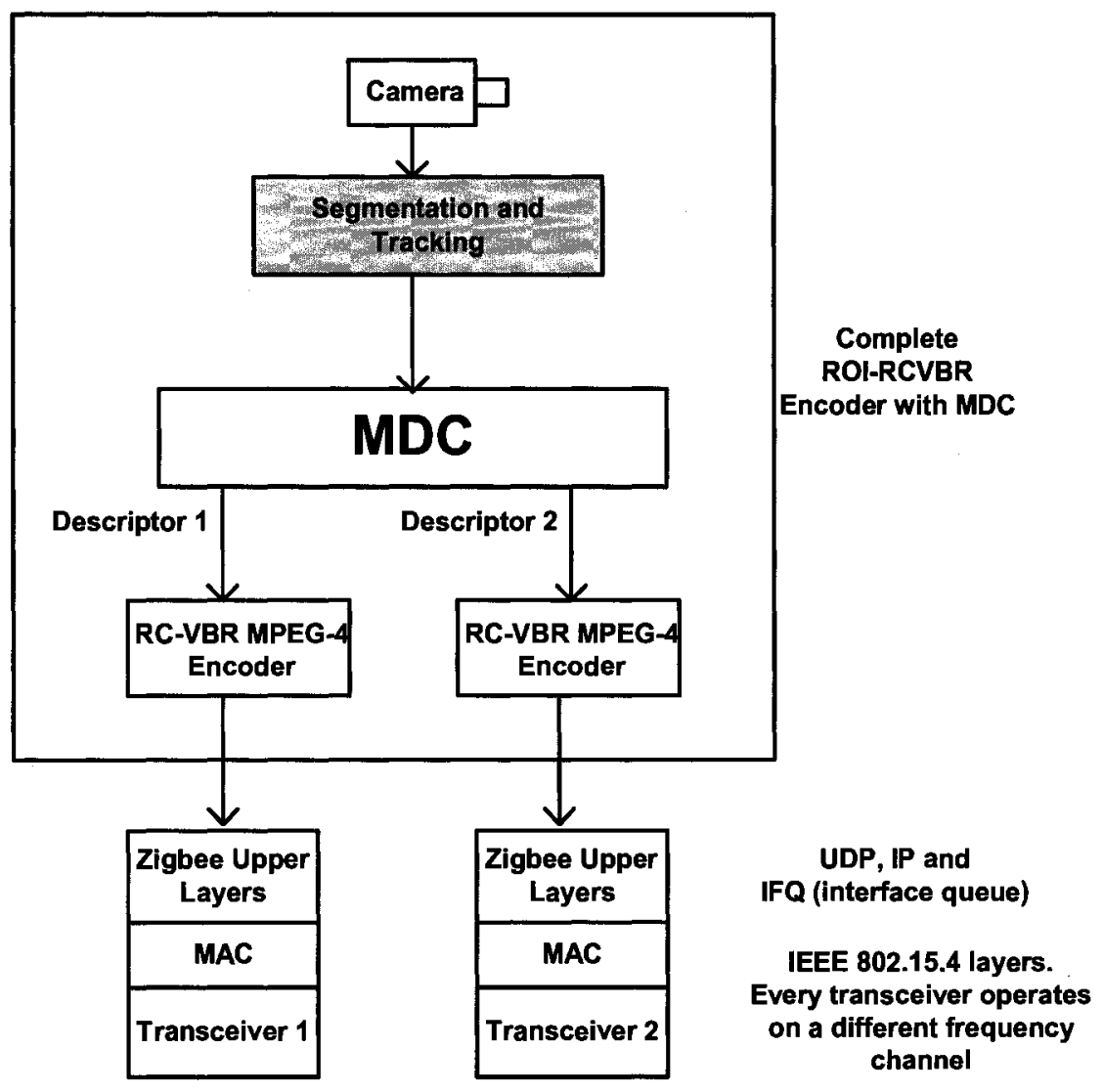

Figure 5.1: Ns Node Structure 


\section{Chapter 6}

\section{Experiments using Simulation Results of Wireless Zigbee Networks}

With the rapidly growing market for short range wireless communication systems, the support of security and surveillance related applications over sensor networks is becoming important. However, many challenges limit the design of efficient video communications over wireless sensor networks. Some of the challenges are due to resource limitations such as limited power and processing capability and the error resilience capability of video compression techniques. In addition, the capacity and throughput provided by wireless channels is much lower than wired channels because of the path loss, fading and interference. Wireless Zigbee networks have additional challenges that limit the capability of video support. The $2.4 \mathrm{GHz}$ band provides the highest theoretical bit rate of $250 \mathrm{Kbps}$ which is low for a large surveillance area. The physical layer supports transfer of only small sized packets limited to 127 bytes. Due to the physical, MAC and upper layer overhead, each has a maximum of 72 bytes for application data. This means that video frames might be fragmented into a higher number of packets depending on the frame type. In addition, the networking layer does not perform fragmentation which means that the fragmentation and reassembly should be handled at the application layer. 
Two simulation metrics are used throughout the simulation: QoS metrics and Subjective metrics. The QoS metrics used are the frame loss percentage and average delay, while the mean opinion score (MOS) is the subjective metric. While the peak signal-to-noise ratio (PSNR) is not a subjective metric, there is a direct mapping from the PSNR metric to mean opinion score (MOS) [38] as shown in Table 6.1.

The PSNR is calculated as follows:

$$
\operatorname{PSNR}(s, d)=20 \log \frac{V_{\text {peak }}}{\operatorname{MSE}(s, d)}[d B]
$$

$V_{\text {peak }}=2^{k}, \mathrm{k}$ is the bit color depth

$\operatorname{MSE}(s, d)=$ Mean Square Error of $\mathrm{s}$ and $\mathrm{d}$

$\mathrm{s}$ (source frame) and $\mathrm{d}$ (destination frame)

Table 6.1: PSNR to MOS mapping

\begin{tabular}{|l|l|}
\hline$P S N R(d B)$ & $M O S$ \\
\hline$>37$ & 5 (Excellent) \\
$31-37$ & 4 (Good) \\
$25-31$ & 3 (Fair) \\
$20-25$ & 2 (Poor) \\
$<20$ & 1 (Bad) \\
\hline
\end{tabular}

The PSNR metric is calculated for every transmitted and received frame. An evaluation metric could also be the average PSNR which takes the mean PSNR of all transmitted and received frames.

This chapter presents the simulation and performance results of the various techniques studied in previous chapters. All simulations are done using the NS-2 simulator which uses $\mathrm{C} / \mathrm{C}++$ and $\mathrm{Tcl}$ for coding.

Throughout the simulations, losses due to the wireless channel are eliminated because of overhead. In addition, each data point collected is averaged over 5 simulation 
trials using a different random seed for each TES model generated.

\subsection{Validity of TES models over wireless Zigbee networks using simulation}

Ns-2 [26] simulations are performed using both real MPEG4 video traces and TES models over wireless Zigbee networks [24]. The purpose is to validate the use of TES models over Zigbee networks. The video sequence consists of 415 frames in QCIF format, each of which is fragmented into a number of Zigbee packets of 127 bytes each (including the overhead). The GOP is IBBPBBPBBPBBI.. which consists of 12 frames. All video frame statistics can be found in table 3.1. It can be shown from figure 3.1 that on reaching a certain number of frames, a peak occurs. This peak represents an I frame. I-frames are much larger than $\mathrm{P}$ and $\mathrm{B}$ frames and are fragmented into a higher number of packets. Therefore, they need more overhead and if some of the I-frame packets are lost, the whole frame and hence the whole GOP is dropped.

Zigbee networks allow single and multi-hop network topologies. In multi-hop (ad hoc) transmissions, the covering range is much higher than the single hop transmissions. However, the throughput is lowered whenever a hop is increased, which results in a lower video quality [27]. This is because of hidden and exposed terminal problems, resulting from the inability of adjacent nodes that are within sensing range of each other to transmit simultaneously. The simulations consider one and two hop transmissions with chain topologies in which losses only occur because of full buffers in the Zigbee nodes.

Figure 6.2 shows the frame loss probability of the real video trace and the video model (generated over 5 trials) of the full video sequence. To understand the queuing 


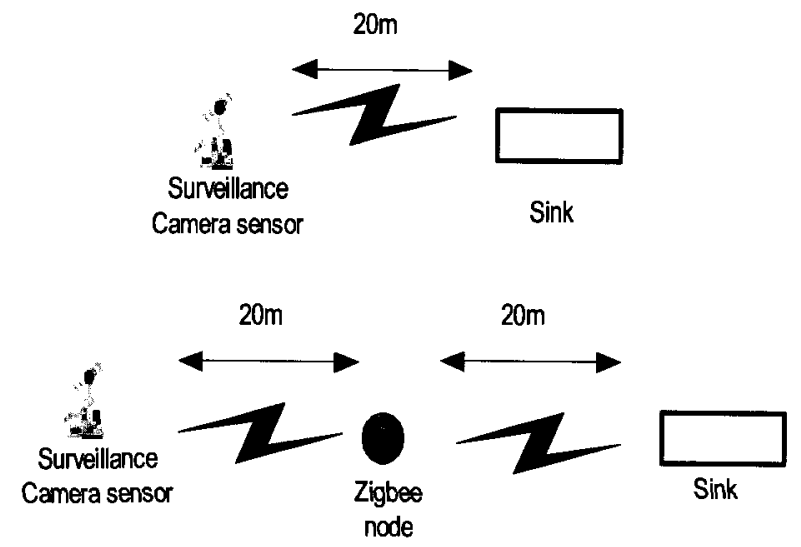

Figure 6.1: a) One hop Zigbee video transmission. b) Two hop Zigbee video transmission.

performance, the buffer size of the Zigbee nodes were increased from 50 to 450 in 50 packet steps. The results in the graph include the one and two hop cases as shown in figure 6.1 with $\mathrm{Q}=4$ and 7 respectively. As shown in the figure, the video model generated closely matches the real trace and as the buffer size increases the frame loss decreases. The values of the quantization parameter were changed for the different number of hop counts so that losses would occur in all cases. Packet losses will only occur because of full buffers. Losses due to the wireless channel will be eliminated because link layer acknowledgments are used.

Figure 6.3 shows the average delay of the video frames for the different quantization parameters. The results include the real video and modeled video. The buffer size was assumed to be high in all the cases so that no frame losses would occur. The figure includes the one and two hop cases. From figure 6.3, we consider the delay of the real video trace and video model to have reasonable agreement. In addition, the delay becomes higher as we increase the number of hop counts. 


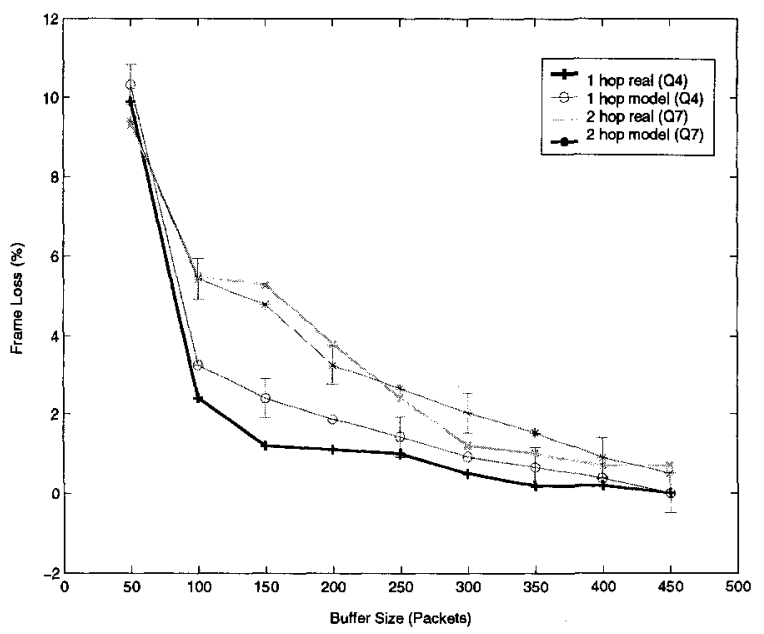

Figure 6.2: Frame Loss vs. Buffer size (bytes)

\subsection{Simulation results of $\mathrm{RC}-\mathrm{VBR}$ algorithm over wireless Zigbee networks}

Ns-2 simulations are performed to compare the RC-VBR algorithm and to show how it outperforms the VBR coding over wireless Zigbee networks. It is very important to choose the right parameters for the RC-VBR algorithm. For example, $b$ defines the buffer size used for the simulations and $r$ is the output buffer rate which directly impacts the video quality. For a reasonable video quality, $r$ should be set to a value greater than $30 \mathrm{~kb} / \mathrm{s}$.

The aggregation node as shown in figure 6.4 is a more complex node which aggregates data from different video sources to a single path and then to the destination. The aggregating node is connected to a power source. Hence, the transmission range is set to a higher value. The destination is the monitoring node where all videos are tracked and any suspicious act can be detected.

The sensing and transmission power is set according to the two-ray-ground model as follows: 


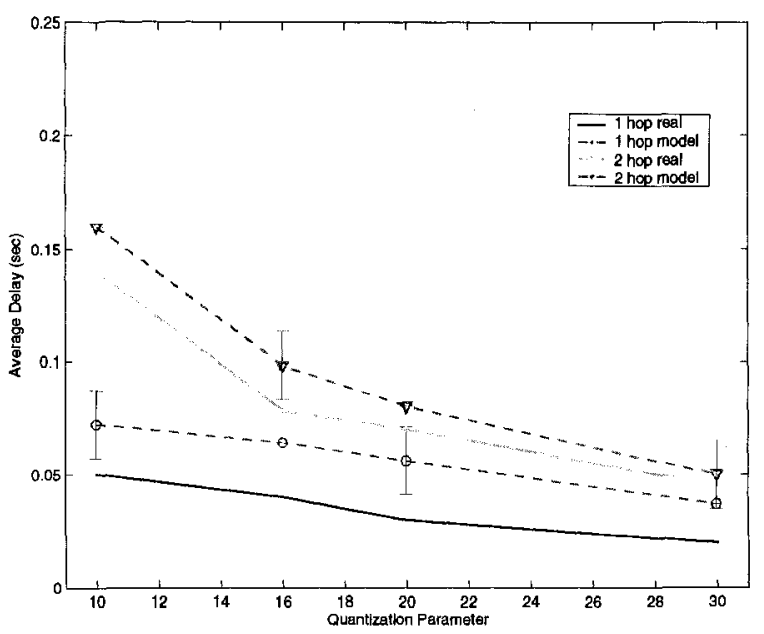

Figure 6.3: Mean Delay(sec) vs. Quantization Parameter

$$
P_{r}=\frac{P_{t} \cdot G_{t} \cdot G_{r} \cdot h_{t}^{2} \cdot h_{r}^{2}}{L \cdot d^{4}}
$$

where $P_{r}$ and $P_{t}$ are the receiving and transmitting powers, $G_{t}$ and $G_{r}$ are the antenna gains, $h_{t}$ and $h_{r}$ are the antenna heights, $L$ and $d$ are the path loss component and transmission distance respectively. This means that in order to support a low transmission power, the transmission distance has to be small.

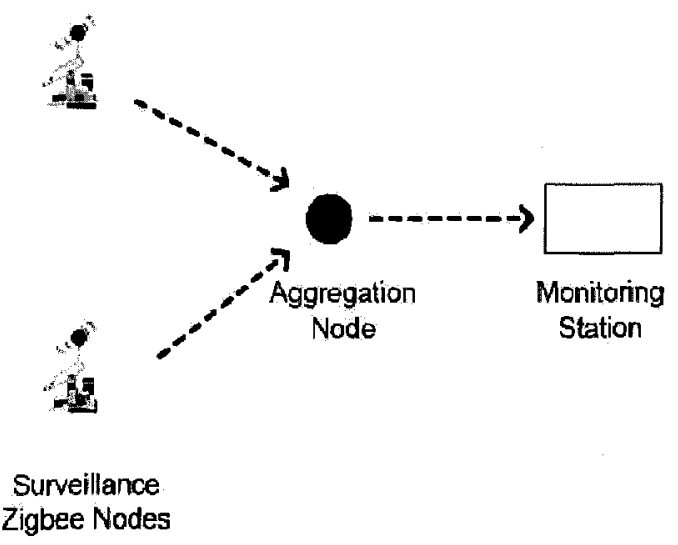

Figure 6.4: Network Topology of the RC-VBR algorithm 
In order to choose the correct parameters for the RC-VBR algorithm, the throughput of the network topology should be known. Although Zigbee networks offer a nominal rate of $250 \mathrm{~kb} / \mathrm{s}$ (i.e. $240 \mathrm{pkts} / \mathrm{sec}$ ), this data rate will be affected by the overhead and number of hop count.

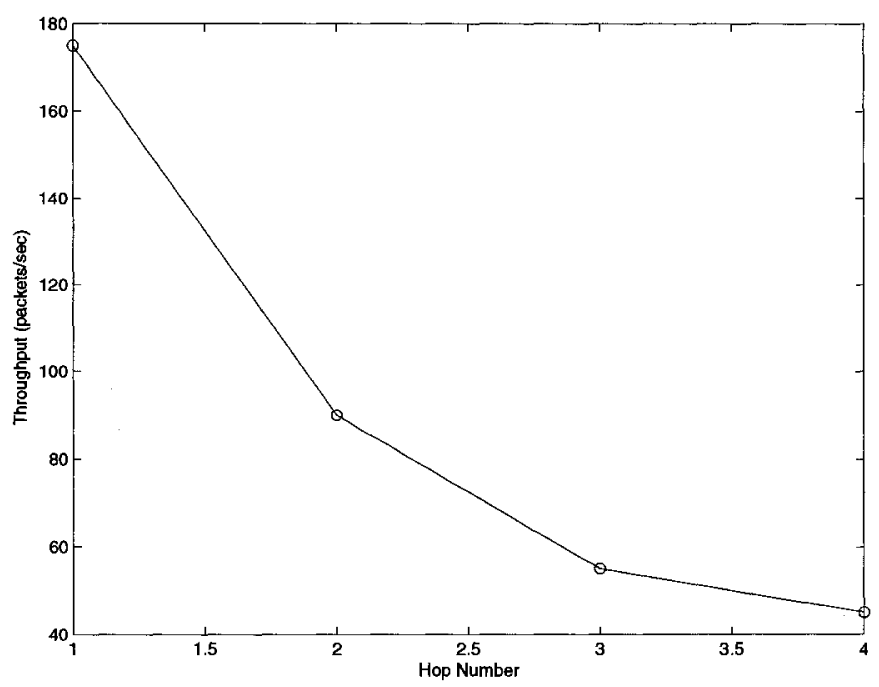

Figure 6.5: Number of hops vs. Throughput (packets/sec)

Figure 6.5 shows the throughput in packets/sec for 1, 2, 3 and 4 hops. It is obvious that the data rate will keep on decreasing drastically up to a certain number of hop counts ( 3 in this case) because of the hidden and exposed terminal problems. After a certain point, the change in the data rate is small because the effects of the terminal and exposed station problems become minimal [27].

From figure 6.5, it is shown that the throughput for the two hop case (the case for figure 6.4 ) is around $75 \mathrm{pkts} / \mathrm{sec}$. This means that in order to get a good video quality over the Zigbee network, we can provide a maximum of two video sources, each with about a $35 \mathrm{~kb} / \mathrm{sec}(35 \mathrm{pkts} / \mathrm{sec})$ rate. Table 6.2 shows all the simulation parameters used in the simulations.

In order to compare VBR with RC-VBR algorithms, a quantization parameter of 15 will be used for the VBR because it produces an average output rate of $35 \mathrm{~kb} / \mathrm{s}$. 


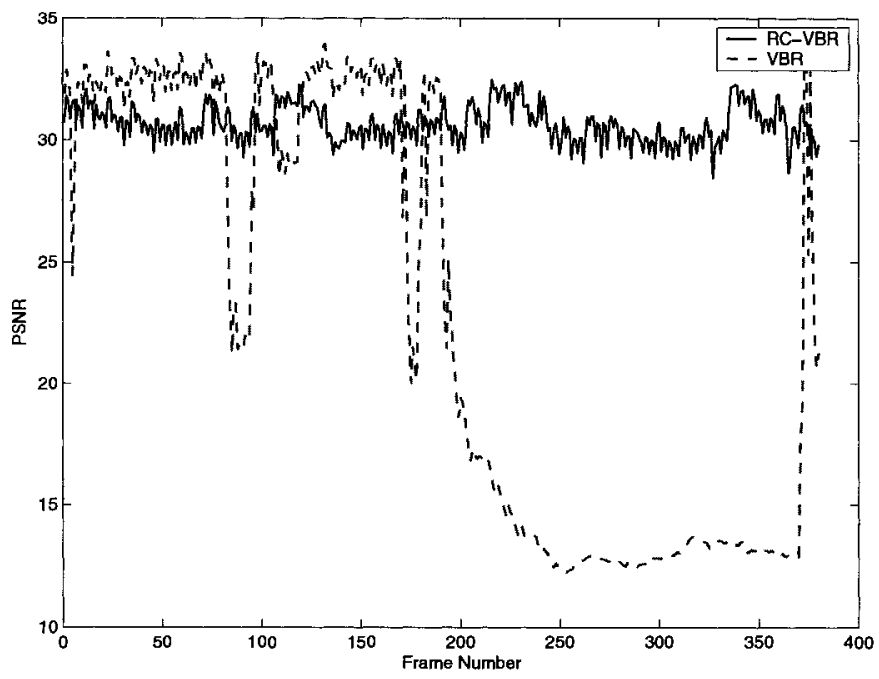

Figure 6.6: Frame Number vs. PSNR

Figure 6.6 shows the PSNR (peak signal-to-noise ratio) using both the VBR and RC-VBR algorithms. The PSNR metric is only shown for one of the video sources while the TES model was fed to the other source. The RC-VBR is implemented in the TES video model using the method shown in figure 3.13 where a scaling factor was used to obtain the frame sizes of the required GOP.

From the figure, it can be shown that RC-VBR algorithm outperforms the VBR algorithm. An important observation is that sometimes (especially at the beginning), the PSNR of the VBR encoding is higher than the RC-VBR. This is because the buffer is not initially full, hence packets can be queued without any loss. This difference is

Table 6.2: Simulation Parameters

\begin{tabular}{|l|l|}
\hline Parameter & Value \\
\hline $\mathrm{b}$ & 50 packets \\
$\mathrm{r}$ & 35 packets $/ \mathrm{sec}$ \\
$d_{s}$ (sources transmission distance) & 20 meters \\
$d_{a}$ (aggregating nodes transmission distance) & 80 meters \\
$\mathrm{x}-\mathrm{y}$ topology coordinates & $100 \mathrm{~m} \times 100 \mathrm{~m}$ \\
\hline
\end{tabular}



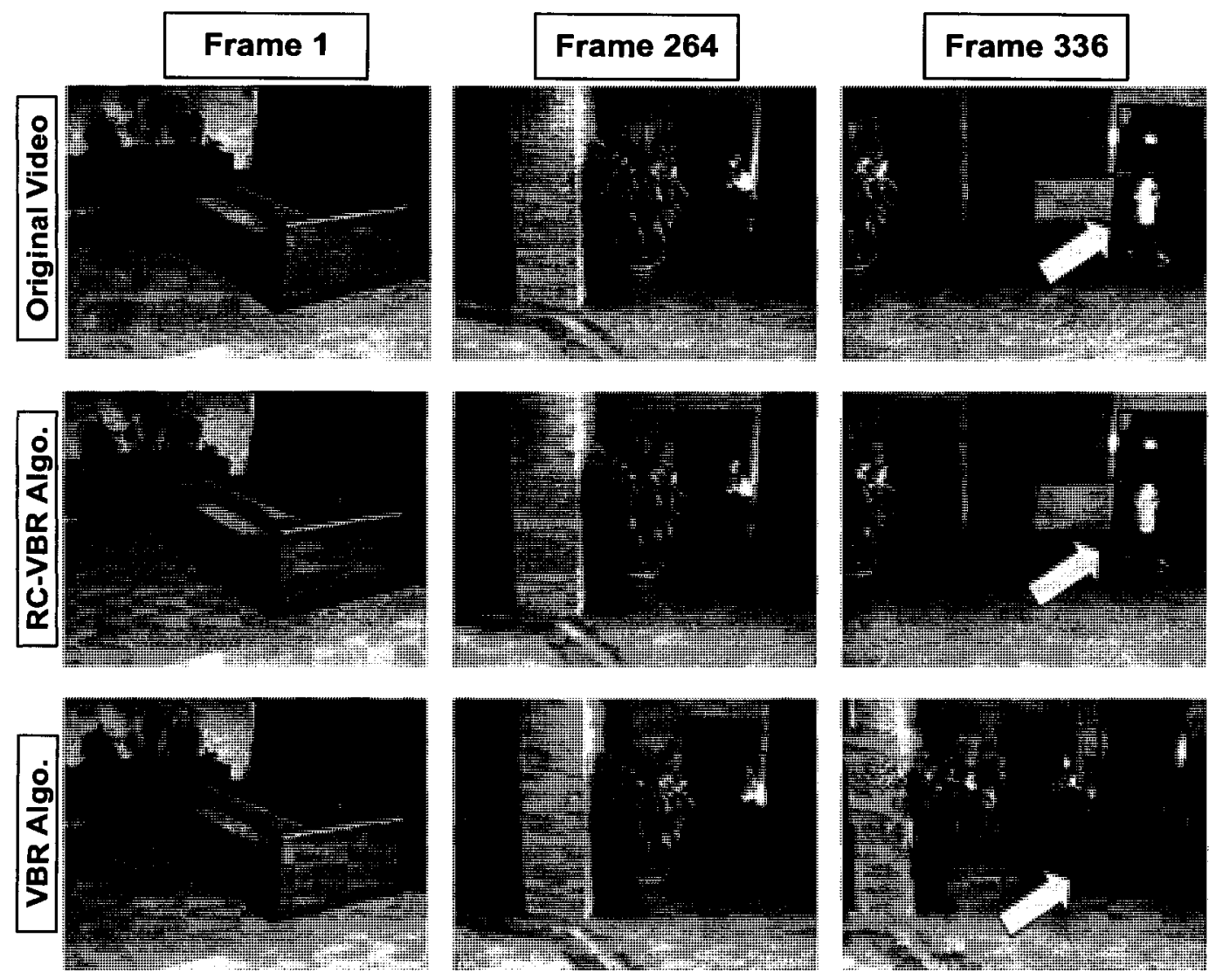

Figure 6.7: Comparison of i)Real video ii)RC-VBR algorithm and iii)VBR encoded sample frames

small and is not obvious. However, as the video sequence continues playing, the VBR encoding starts to loose a lot of packets because of the rate variability that it offers. Whereas in the RC-VBR case, the algorithm makes sure that the buffer occupancy stays within certain boundaries.

Figure 6.7 shows a comparison between some parts of the real video, RC-VBR and VBR received videos. From figure 6.7, the conclusions that were made concerning figure 6.6 can be proven visually (by looking at the arrows where the object cannot be observed). This shows that RC-VBR outperforms VBR for Zigbee networks.

To validate the previous results, 4 more video samples were taken for the same 
topology in figure 6.4. The average PSNR and frame loss percentage are taken as the evaluation metrics. Table 6.3 shows the comparison of the video samples. The table shows that RC-VBR outperforms the VBR algorithm in all the cases. Figure 6.8 validates the above results by showing the real video, the RC-VBR algorithm and the VBR encoding respectively.

Table 6.3: Comparison of the performance metrics of VBR and RC-VBR algorithms

\begin{tabular}{|l|l|l|l|l|}
\hline & \multicolumn{2}{|l|}{ RC-VBR algorithm } & \multicolumn{2}{l|}{ VBR algorithm } \\
\cline { 2 - 5 } & $\begin{array}{l}P S N R \\
(d B)\end{array}$ & $\begin{array}{l}\text { Frame } \\
\text { Loss }(\%)\end{array}$ & $\begin{array}{l}\text { PSNR } \\
(d B)\end{array}$ & $\begin{array}{l}\text { Frame } \\
\text { Loss(\%) }\end{array}$ \\
\hline \hline Walking Person & 29.21 & 0.0 & 23.67 & 9.2 \\
Skater & 29.46 & 0.0 & 23.54 & 30.4 \\
Skier & 32.03 & 0.0 & 30.56 & 7.1 \\
Soccer Player & 26.37 & 0.0 & 24.29 & 26.1 \\
\hline
\end{tabular}



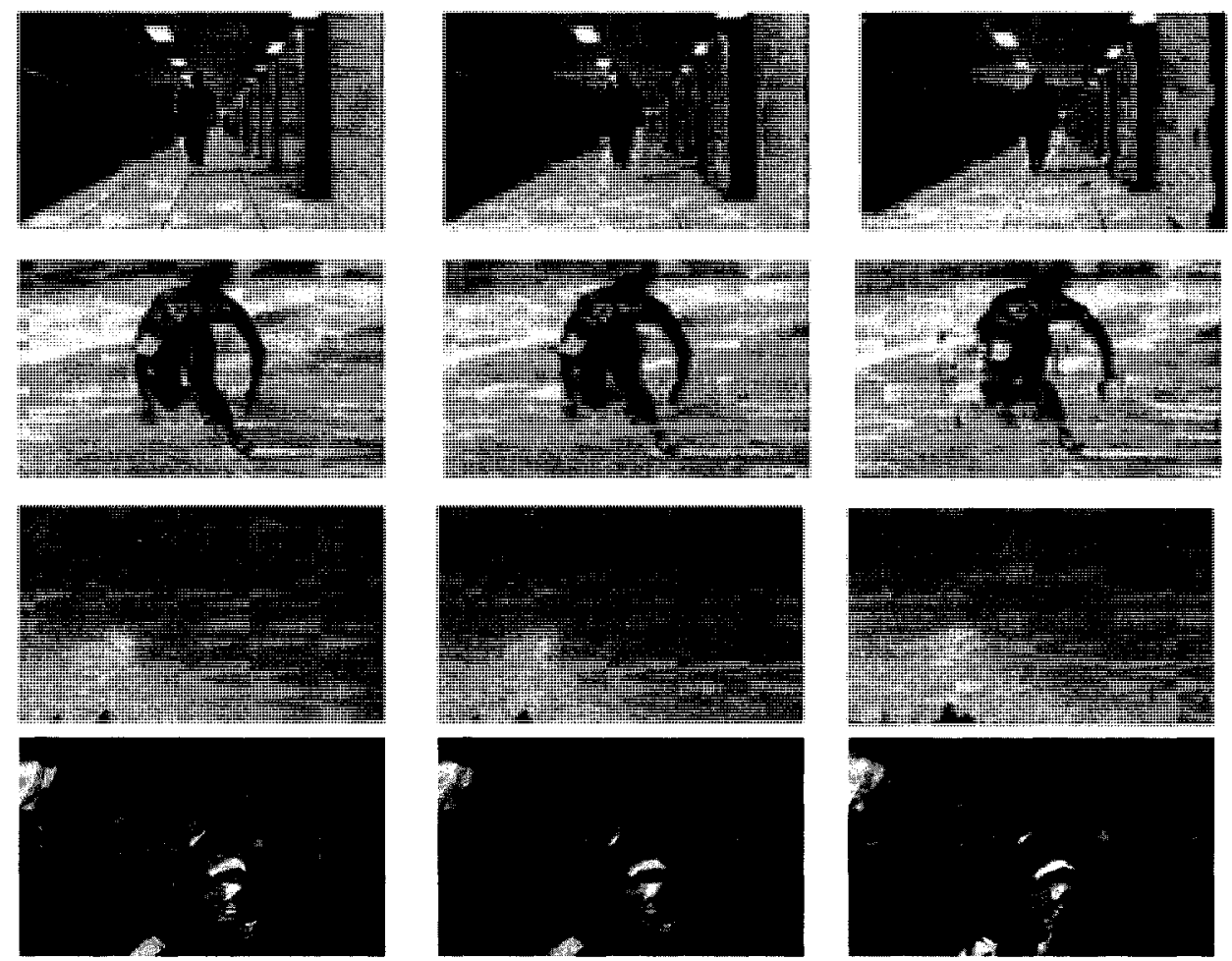

Figure 6.8: i) Real video ii) RC-VBR algorithm iii) VBR encoded sample frames

\subsection{Simulation results of ROI RC-VBR algorithm over wireless Zigbee networks}

Although RC-VBR produced good results, the maximum number of sources that were accommodated were small (two in our case) since IEEE 802.15.4 networks offer a very low data rate. Therefore, a combination of region-of-interest (ROI) coding with a source rate control VBR (RC-VBR) encoding is used, as developed in section 4.3. This is done by dynamically adapting encoding parameters for the region of interest in the different video frames. Hence, the number of video surveillance cameras that 
can be accommodated with a low loss rate over the Zigbee network are increased to four sources.

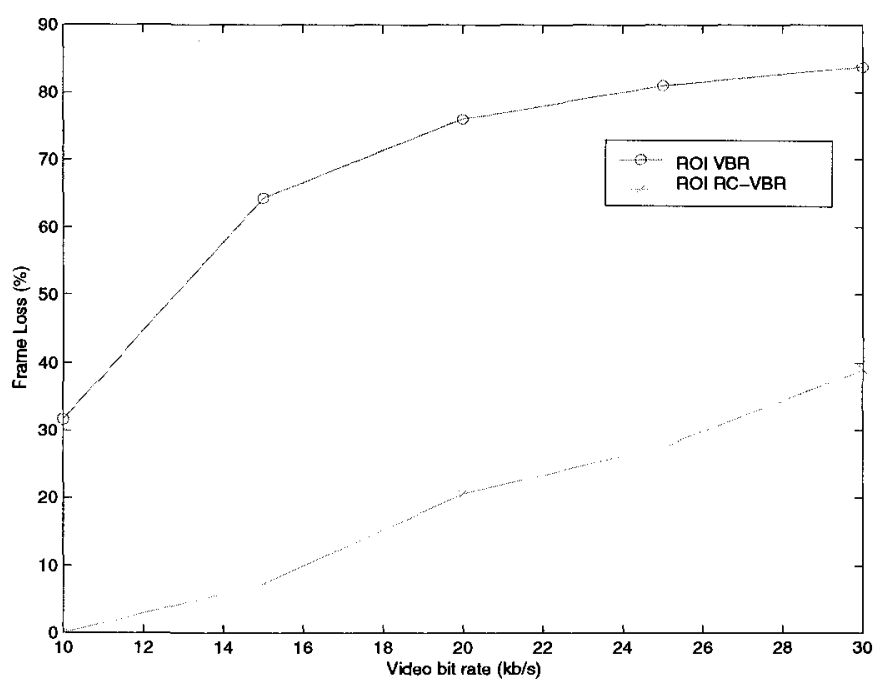

Figure 6.9: Video bit-rate vs. Frame Loss

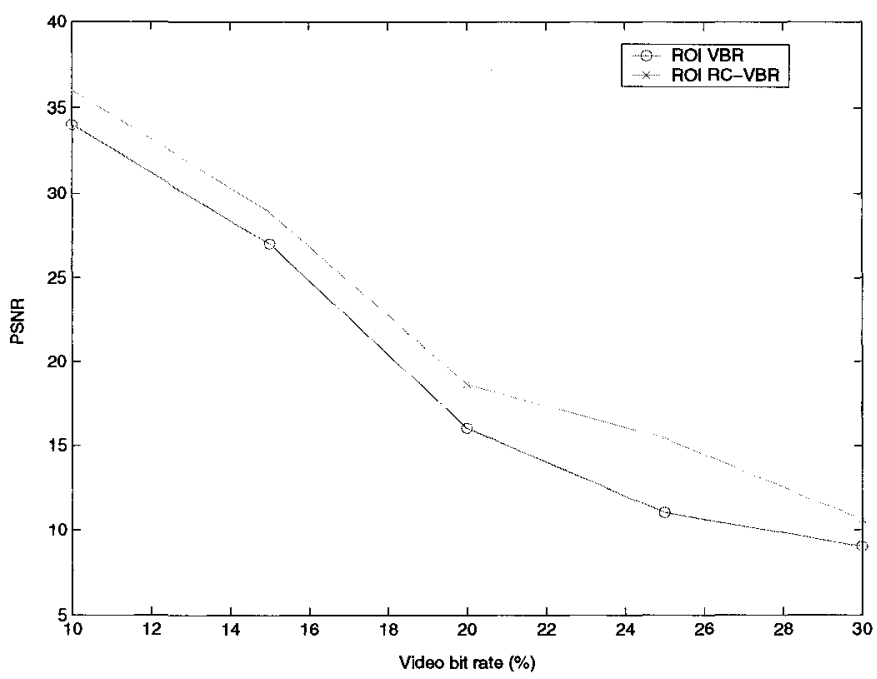

Figure 6.10: Video bit-rate vs. Average PSNR

Table 6.4 shows the average PSNR and frame loss percentage of the ROI-RCVBR, RCVBR and ROI-VBR Zigbee video transmissions when four video transmissions are used. By comparing the ROI-RCVBR and RCVBR algorithms, the addition of the 
region-of-interest algorithm results in improved performance, increasing the topology size of the video transmission over Zigbee networks.

Figures 6.9 and 6.10 illustrate a comparison between ROI-RCVBR encoding and ROI-VBR encoding using 4 video sources (walking person is used in this case). From the figures, the ROI-RCVBR algorithm resulted in an average decrease of $40 \%$ in the frame loss and an average increase of $2.5 \mathrm{~dB}$ in the average PSNR. An important observation is that with ROI-RCVBR, a $10 \mathrm{~kb} / \mathrm{s}$ video bit rate produces $0 \%$ loss and very good video quality. This is because only important parts of the frames are transmitted, which means that this bit rate is suitable for wireless Zigbee networks.

Table 6.4: Comparison of the performance metrics of ROI-VBR, RCVBR and ROIRCVBR algorithms with 4 video sources

\begin{tabular}{|l|l|l|l|l|l|l|}
\hline & \multicolumn{2}{|l|}{ ROI-RCVBR algorithm } & \multicolumn{2}{l|}{ RCVBR algorithm } & \multicolumn{2}{l|}{ ROI-VBR algorithm } \\
\cline { 2 - 7 } & $\begin{array}{l}P S N R \\
(d B)\end{array}$ & $\begin{array}{l}\text { Frame } \\
\text { Loss(\%) }\end{array}$ & $\begin{array}{l}P S N R \\
(d B)\end{array}$ & $\begin{array}{l}\text { Frame } \\
\text { Loss(\%) }\end{array}$ & $\begin{array}{l}\text { PSNR } \\
(d B)\end{array}$ & $\begin{array}{l}\text { Frame } \\
\text { Loss(\%) }\end{array}$ \\
\hline \hline Walking Person & 35.96 & 0.0 & 25.53 & 21.1 & 34.20 & 32.0 \\
Skater & 27.03 & 17 & 20.2 & 33.2 & 20.01 & 71.7 \\
Skier & 35.0 & 0.0 & 24.56 & 20.8 & 22.67 & 56.0 \\
Soccer Player & 26.55 & 18.4 & 18.74 & 35.3 & 21.29 & 71.3 \\
\hline
\end{tabular}

To validate the efficiency of the ROI-RCVBR algorithm, the same 4 video samples are taken. Table 6.4 also shows the average PSNR and frame loss percentage of the ROI-RCVBR and the ROI-VBR Zigbee video transmissions. It can be shown that the ROI-RCVBR algorithm results in improved performance, enabling the transmission of video over a Zigbee environment. From table 6.4, it can be observed that the ROI-VBR performs the worst among all three algorithms. This proves that the ROI algorithm is not enough to increase the topology size of the network if VBR coding is used without adding the rate control algorithm.

An important observation from the table is the difference in quality among the 
video sequences. For instance, the walking person and skier vidoe sequences produce the best performance when using the ROI-RCVBR algorithm. This is because the segmented video object uses the smallest portion of the original video frame. 


\subsection{Effects of interference of WLANs (IEEE 802.11) and LR-WPANs (IEEE 802.15.4/Zig- bee) on video surveillance LR-WPANs}

This section will illustrate the effect of interference in WPANs caused by both WPANs and WLANs in a wireless surveillance application. For all the experiments, the transmission range of the Zigbee devices is 20 meters while the range for the WLAN devices is 250 meters.

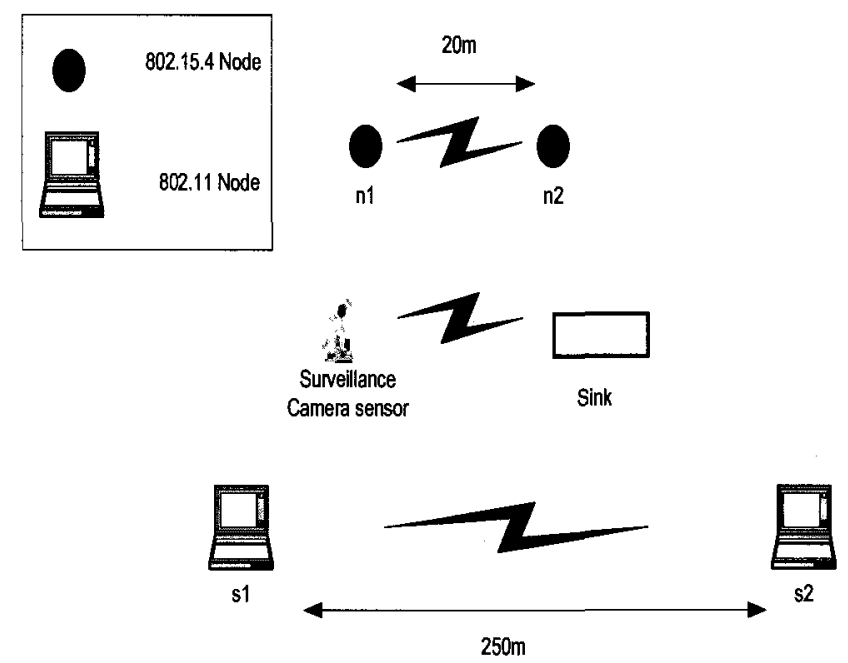

Figure 6.11: LR-WPAN and WLAN interference topologies

To study the effect of interference between low bit-rate WPANs and WLANs, we refer to figure 6.11. The two experiments shown in the figure are performed separately. The surveillance camera sensor is the Zigbee video source that is outputting an MPEG-4 encoded video to a sink with a quantization parameter (Q) 10. Nodes $\mathrm{n} 1$ and $\mathrm{n} 2$ are two Zigbee nodes that are sending data at a constant bit rate (CBR) distribution with a packet size of 100 bytes and inter-arrival time of $10 \mathrm{~ms}$. Both networks are using the same frequency channel to study the effect of interference. 
Table 6.5 shows the results of the effect of interference that occurs between the two IEEE 802.15.4 transmissions. The results show the frame loss percentage and average PSNR in the presence of another IEEE 802.15.4 network.

A separate experiment was done and also shown in figure 6.11. The same surveillance camera sensor and Sink are used in the experiment. The IEEE 802.11 network consists of nodes s1 and s2. Node s1 transmits CBR traffic with packet size 512 bytes over a UDP connection. The WLAN network uses a channel that interferes with that of the WPAN video surveillance network. The frame loss and average PSNR is calculated and also shown in table 6.5. From table 6.5, it can be shown that the coexistence of IEEE 802.15.4 and WPAN networks has a great effect on video transmission. However, the IEEE 802.11 has a greater effect on video quality because it uses higher power, wider frequency spectrum and greater packet sizes than the IEEE 802.15.4 network which conforms to the previous work.

Table 6.5: Impact of interference on IEEE 802.15.4 networks

\begin{tabular}{|l|l|l|}
\hline & FrameLoss $(\%)$ & $P S N R(d B)$ \\
\hline WPAN Interference & 39.7 & 25.11 \\
WLAN Interference & 53.2 & 20.21 \\
\hline
\end{tabular}




\subsection{Simulation study of multi-channel multi-radio over wireless Zigbee networks}

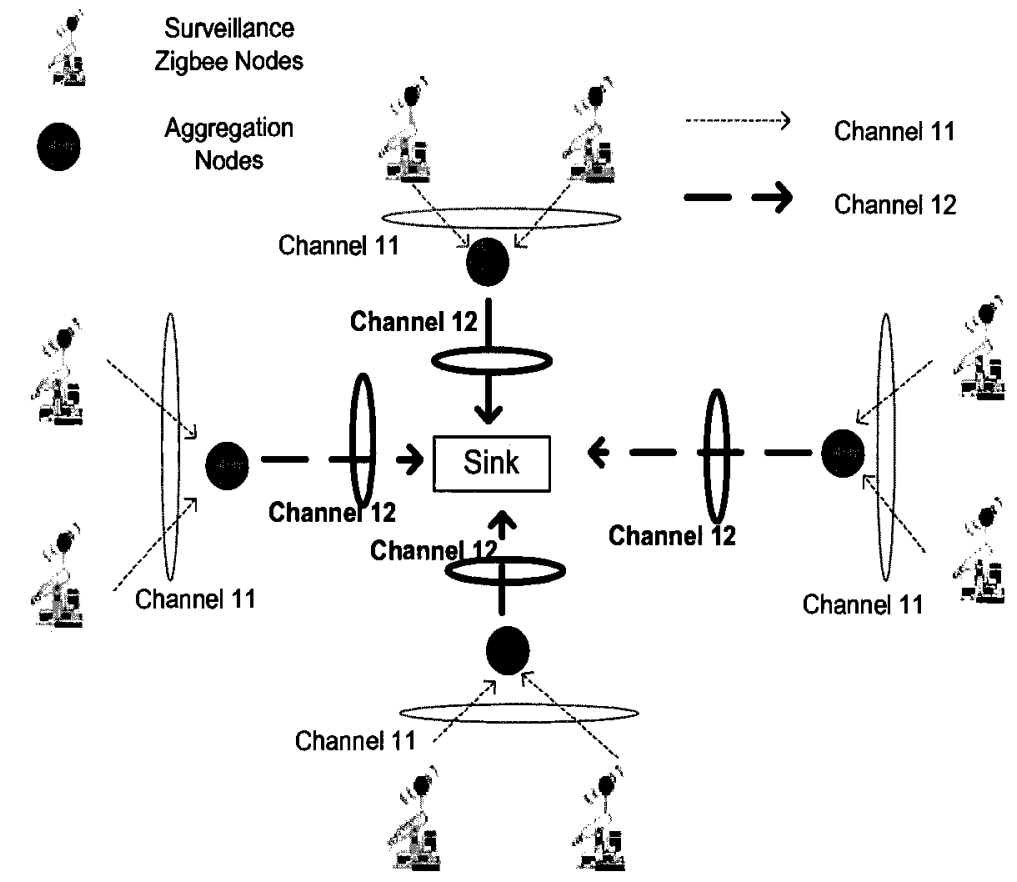

Figure 6.12: Network Topology with 2 channels and 2 interfaces

The original network topology used before in section 6.5 consisted of a topology where all nodes used one interface and one channel for the whole network. The number of video sources was limited to 4 even though the region-of-interest (ROI) video encoding was used. Therefore, multi-channels and multi-interfaces are used to increase the topology size. Figure 6.12 is similar to the one used in section 6.5 . However, the aggregation nodes are equipped with 2 interfaces where each interface uses a different channel. Since the $2.4 \mathrm{GHz}$ ISM band in the IEEE 802.15.4 standard contains 16 channels, we may assign two different channels to the two interfaces, for instance channels 11 and 12. All Zigbee video sources are equipped with one interface 
and hence use a single channel, i.e. 11. The sink node will only need a single interface since only 2 channels are used by the topology. Therefore, the sink node will use the second channel, 12 for our case, to communicate with the other aggregation nodes. This network topology will increase the throughput by a factor of 2 , which means that the number of ROI-RCVBR video sources is doubled, i.e. becomes 8 in our case. This is shown in table 6.6 where 7,8 and 9 sources are assumed. For each case the frame loss percentage and the PSNR are calculated for source 1 (the real video source), while TES models are being used for the rest of the video sources. It is shown that no loss occurs in the 7 and 8 source cases, whereas a loss of $8.1 \%$ occurs in the 9 sources case as expected. An important observation is that no quality difference in the video received for the 7 and 8 source cases since all sources will be accommodated in both cases.

Table 6.6: Effect of number of sources on a 2 channel and a 2 interface topology

\begin{tabular}{|l|l|l|}
\hline Numberof Sources & FrameLoss $(\%)$ & $P S N R(d B)$ \\
\hline 7 & 0 & 35.96 \\
8 & 0 & 35.96 \\
9 & 8.1 & 33.5 \\
\hline
\end{tabular}

This network topology can be further enhanced by using more channels or interfaces per node. However, as mentioned in related studies, a maximum of 2 interfaces are used per node because more power is needed which is not suitable for Zigbee nodes. Therefore, if the number of channels is increased, more sources can be handled. For example, for the topology shown in figure $6.13,12$ sources can be used. As illustrated in the figure, the aggregation nodes and the sink node use 2 interfaces. The surveillance nodes still use a single interface. However, the different aggregation nodes will not interfere with each other since they are not within interference range. Table 6.7 shows the frame loss percentage and average PSNR for 11,12 and 13 video 
sources. Similarly, a $0 \%$ loss and the same PSNR occurred for the 11 and 12 source cases while a loss of $5.6 \%$ and a lower PSNR occurred for the 13 sources case as expected. The use of more channels will result in an increase in the throughput and hence in the number of video sources. In addition, even if more channels are available in the network which will most likely be the case for the $2.4 \mathrm{GHz}$ in the IEEE 802.15.4 standard, the throughput can be increased to accommodate more sources.

Table 6.7: Effect of number of sources on a 3 channel and a 2 interface topology

\begin{tabular}{|l|l|l|}
\hline NumberofSources & FrameLoss $(\%)$ & $P S N R(d B)$ \\
\hline 11 & 0 & 32.73 \\
12 & 0 & 32.73 \\
13 & 5.6 & 31.26 \\
\hline
\end{tabular}

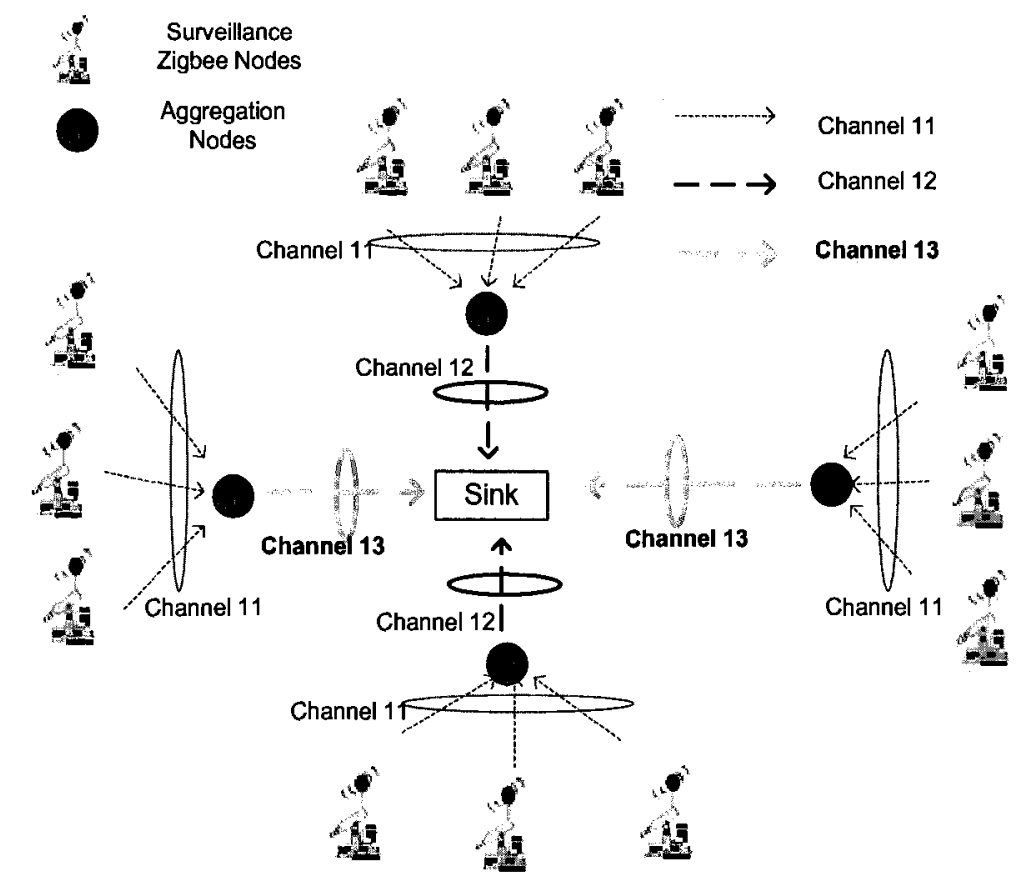

Figure 6.13: Network Topology with 3 channels and 2 interfaces 


\subsection{Network topology and simulations of Multiple Description Coding (MDC) over multi-radio multi-channel Zigbee networks}

The new topology used for the simulations is shown in figure 6.14. All the nodes including the camera sensors are equipped with 2 interfaces. Four channels are used for the entire network as shown in the figure.

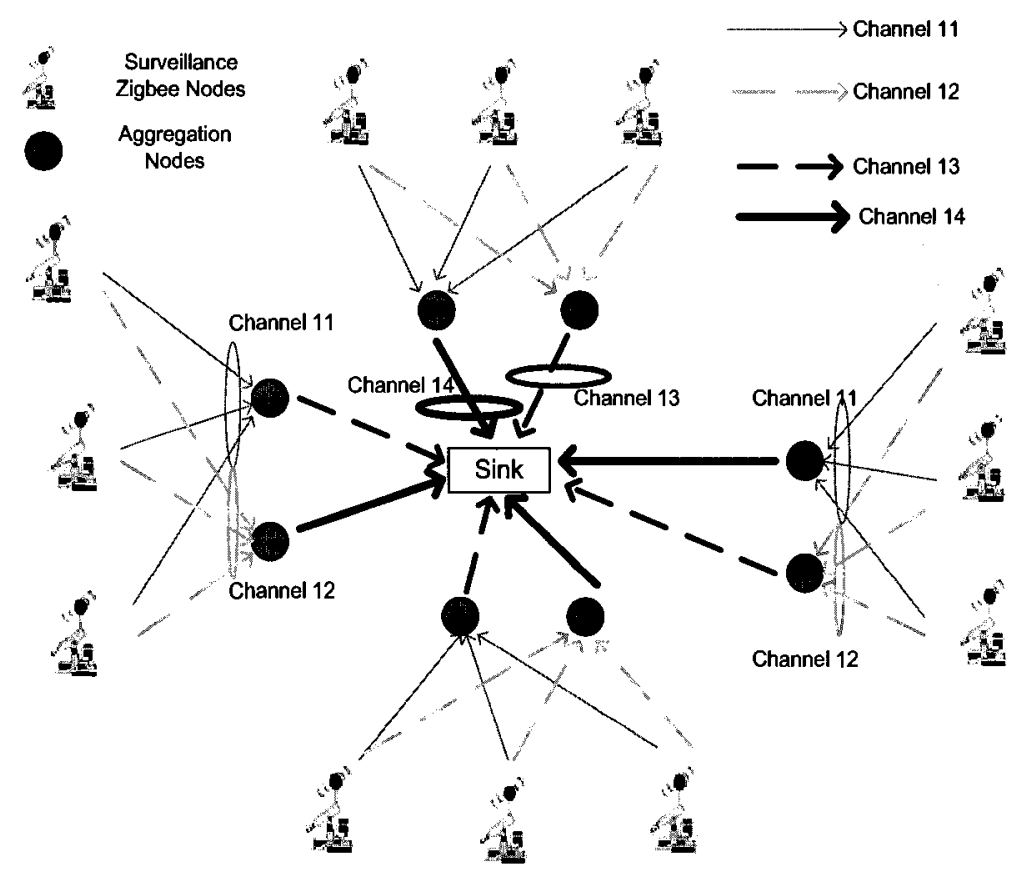

Figure 6.14: Network Topology with 4 channels and 2 interfaces with MDC

As MDC splits the video source stream into multiple descriptors, the splitter will split the stream using a frame based approach. The splitter, as shown in figure 6.15, takes the raw video sequences and splits them into i sub-sequences ( 2 in our case). Then the sub-sequences are fed to the video encoder, MPEG4 in our case.

All previous simulations assumed interference but no channel errors during the 


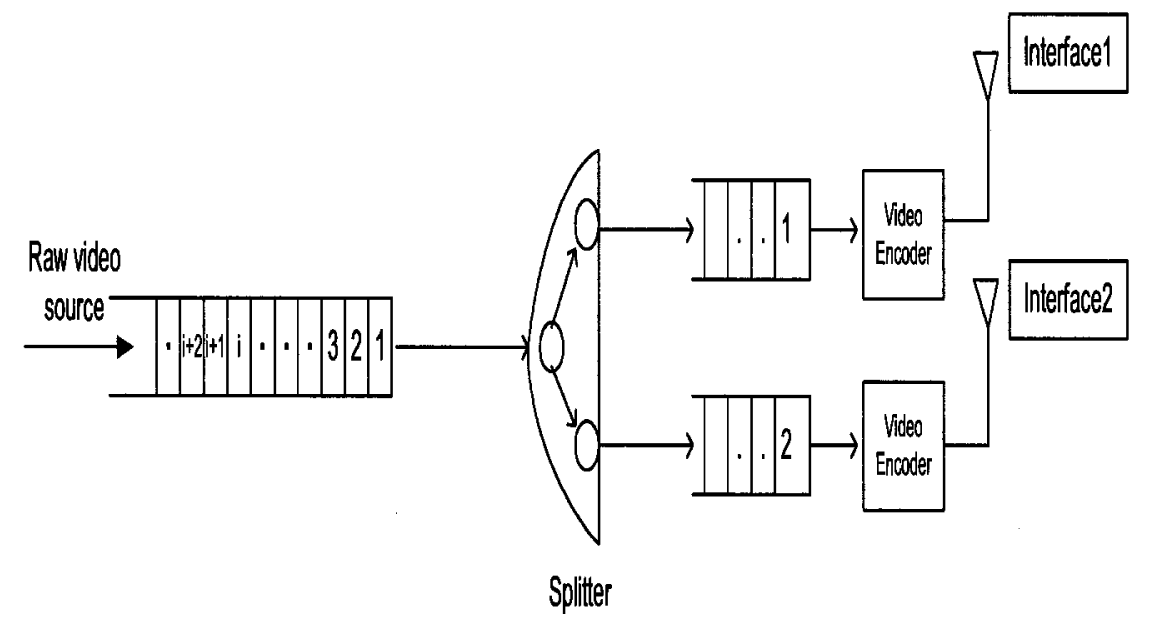

Figure 6.15: Multiple Descriptive Coding (MDC)

transmission. However, in real wireless environments, many errors occur because of fading and other wireless errors. The Gilbert-Eliot error model is chosen to show the efficiency of using MDC with multi-channel multi-interface video transmission [38].

Wireless GE model, also known as burst error model is shown in figure 6.16. It consists of a "good" state $(G)$ where errors occur with lower probability $P_{G}$, while in the "bad" state (B), errors happen with higher probability $P_{B}$. Also, $P_{G B}$ is the probability of the state transiting from a good state to a bad state, and $P_{B G}$ is the transition from a bad state to a good state. The steady state probabilities of being in states $\mathrm{G}$ and $\mathrm{B}$ are

$$
\pi_{G}=\frac{P_{B G}}{P_{B G}+P_{G B}} \text { and } \pi_{B}=\frac{P_{G B}}{P_{B G}+P_{G B}} \text { respectively. }
$$

The average packet loss probability produced by the GE error model is

$$
P_{\text {avg }}=P_{G} \cdot \pi_{G}+P_{B} \cdot \pi_{B}
$$

For simplicity, some researchers assume the random uniform model to represent the error characteristics of a wireless channel. However, a wireless channel has burst 


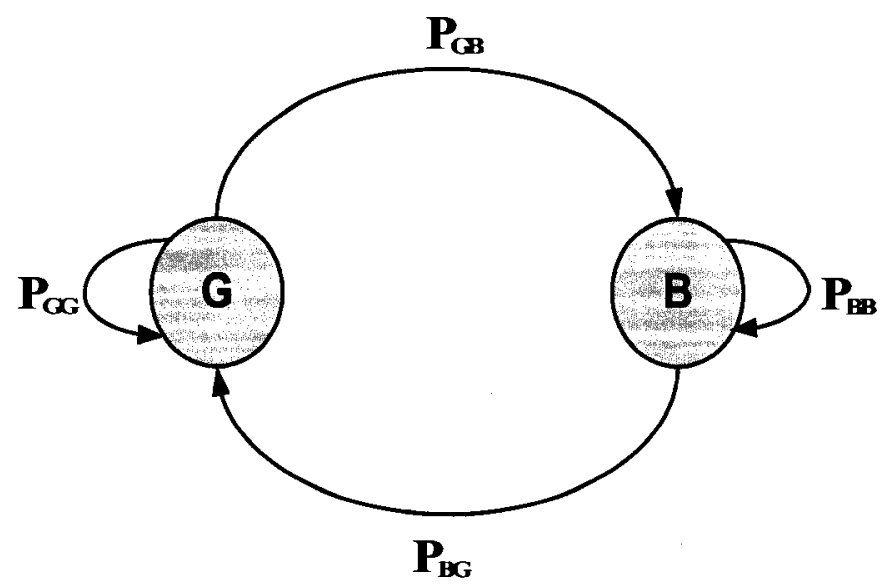

Figure 6.16: Gilbert-Eliot error model

error patterns, and the Gilbert-Eliot model [38] is a well known channel model used to measure the burst error pattern.

Table 6.8: Comparison of the performance metrics of ROI-RCVBR with and without MDC

\begin{tabular}{|l|l|l|l|l|}
\hline \multirow{2}{*}{} & \multicolumn{2}{|l|}{ ROI-RCVB (MDC) } & \multicolumn{2}{l|}{ ROI-RCVBR (no MDC) } \\
\cline { 2 - 5 } & $\begin{array}{l}P S N R \\
(d B)\end{array}$ & $\begin{array}{l}\text { Frame } \\
\text { Loss }(\%)\end{array}$ & $\begin{array}{l}P S N R \\
(d B)\end{array}$ & $\begin{array}{l}\text { Frame } \\
\text { Loss }(\%)\end{array}$ \\
\hline \hline Walking Person & 36.55 & 2.6 & 28.52 & 11.2 \\
Skater & 23.72 & 26.3 & 20.04 & 18.8 \\
Skier & 35.57 & 4.7 & 21.98 & 13.8 \\
Soccer Player & 25.40 & 22.6 & 20.69 & 29.4 \\
\hline
\end{tabular}

The $P_{G G}, P_{B B}, P_{G}$ and $P_{B}$ are set to $0.96,0.94$, and 0.01 , and 0.3 respectively. The parameters are similar to the ones taken in [38]. The average PSNR and the frame loss rate of one of the Zigbee camera sensors are shown in table 6.8 using multi-channel and multi-interface with and without MDC for the walking person, skater, skier and soccer player video sequences [37] respectively. The big difference in the average PSNR and frame loss rate is due to a high error rate that was assigned to 
the GE error model which resulted in high losses in one of the channels. In addition, by using MDC, all of the errors can be restored as long as one of the descriptions is received correctly. Hence, producing the high average PSNR value. The results can be illustrated in figure 6.17 by showing a sample frame from the video using MDC and the video using no MDC. It can be shown in the figure that some parts of the video are not clear without MDC. This is because some errors occurred during transmission because of the channel error model introduced to the wireless channel. This could result in losing important portions of the frame, especially in object based video suveillance. An important observation from the figure is that the different video types are different in quality, even though the results are better for all the video sequences when MDC is used. However, the surveillance sample (walking person) still produced the best results because the object size and movement within the video are the least among all the video sequences. Thus, wireless Zigbee networks can be used for such networks efficiently even with the introduction of channel errors. 

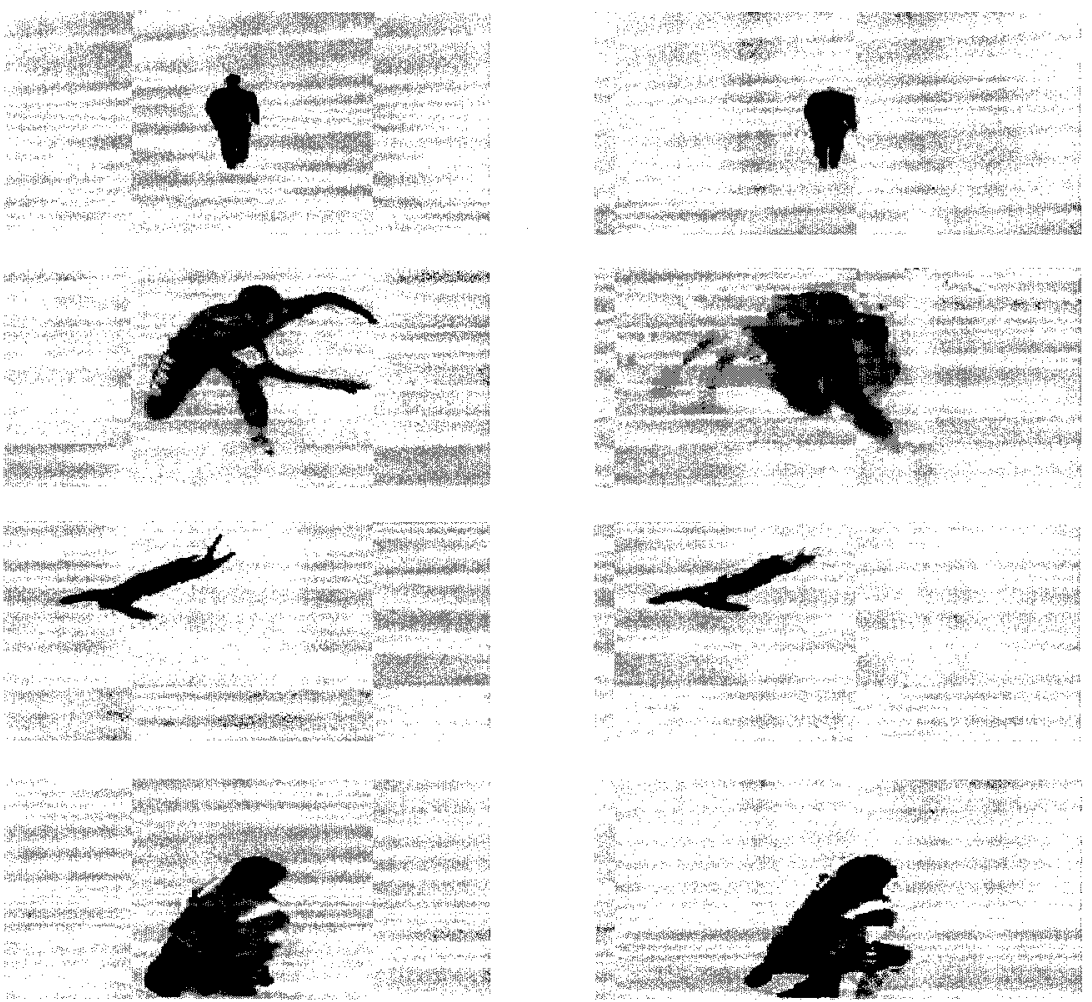

Figure 6.17: Comparison of multi-channel multi-interface using i) with MDC and ii) no $\mathrm{MDC}$ 


\section{Chapter 7}

\section{Conclusions and Future Work}

This chapter contains the conclusions and suggested directions for future study.

ZigBee is a promising technology designed especially to allow low cost and low power communication among devices running within a personal area network (PAN). IEEE802.15.4/ZigBee has been steadily accepted by the industry as a de-facto industry standard; more and more manufacturers produce ZigBee compatible devices.

In this thesis, a rate control video transmission scheme was developed to enable specific video applications over wireless Zigbee networks. The video applications included low rate video surveillance and monitoring transmission rather than high rate video streaming as can be seen with other wireless standards that offer higher data rates. Moreover, a simulation based study for video transmission over distributed nodes based on a multi-channel multi-interface IEEE 802.15.4/ZigBee network was presented.

\subsection{TES modeling for low bit-rate MEPG4 video and future directions}

It was shown that TES processes offer good modeling approach for low bit rate MPEG4 video traffic. The frame types and overall interleaved video model matched 
the original video sequence. The thesis outlined a generation method of video models for the different quantization parameters without having to do all the steps of TES modeling. It is done by modeling the compressed video for a small number of quantization parameters, and then multiplying each frame type by a certain scaling factor that can be different depending on the video type. The scaling factor approach enables the study of video performance over wireless Zigbee networks. Video transmission can occur without the need for transmitting the real video since both the original and modeled traffic provide similar statistics. For future work, more properties of the video trace models may be developed such as generating different priority packets and capturing self-similarity of the video traffic.

\subsection{Summary of implemented topic results and fu- ture work}

The rate control variable-bit-rate (RC-VBR) algorithm was introduced and was shown to outperform video transmission over IEEE 802.15.4 Zigbee networks using traditional techniques. The quantization parameter was changed adaptively for the different video sources by continuously monitoring the buffer occupancy. The performance metrics were calculated for the original MPEG4 source while all other sources were fed by the TES models. A combination of region-of-interest (ROI) and RC-VBR was implemented for improved video surveillance by adding more video sources to the network (a maximum of 4 sources is reached).

The effect of interference of 802.15.4 (LR-WPAN) and 802.11 (WLAN) on a video transmission over wireless Zigbee networks was studied. It was shown that both cases degraded the video quality. However, the interfering 802.11 transmission had a greater impact than the 802.15.4 interfering nodes due to its higher transmission 
power, wider frequency spectrum and greater packet sizes.

A multi-channel multi-radio network topology was extended to work for wireless Zigbee networks. This topology takes advantage of the multiple free non-overlapping channels that are available in the IEEE 802.15.4 standard. The number of sources in the wireless Zigbee network was increased due to the substantial improvement in throughput of the multi-channel multi-radio network. The number of interfaces (i.e. radios) used in the simulations were 2 as the number of channels were being changed. A two channel topology resulted in an increase in the number of video camera sources by a factor of 2 (i.e. peaked at 8 sources) and up to 12 video sources with a three channel topology.

An enhancement of the multi-channel multi-interface topology was developed by adding multiple descriptive coding (MDC) to the ROI-RCVBR algorithm. Using this coding technique, the delivery of the video content was guaranteed even in the presence of channel errors (simulated by the Gilbert-Eliot model) which are frequent in real environments.

All the work in the thesis was implemented in a software simulator (NS-2), which make the topics studied in this thesis less attractive for real-time purposes, i.e. video surveillance. Therefore interesting future enhancements to this research would be the following:

- Implementation of all the studied topics (MDC, ROI and RC-VBR) in Matlab.

- Implementation of all the studied topics in HDL (Verilog or VHDL).

The software application that should be chosen for the developed algorithms should be Matlab. Matlab is a robust tool for the development of PC-based prototypes for targeted hardware platforms. Such implementations are well suited for Matlab since it has strong support for matrix-based computations like image processing. Matlab is also a useful algorithmic tool for the purpose of video and digital signal processing. 
As the majority of the image processing algorithms are highly recursive tasks, the hardware candidate technologies for implementation are DSP (Digital Signal Processor), FPGA (Field Programmable Gate Arrays) and ASIC (Application Specific Integrated Circuit). The prime choice for the implementation design of the algorithms is FPGAs since it has the best performance and most feasible cost. DSP technology is no different from a general-purpose computer, because of its sequential nature. ASIC on the other hand can be expensive for low-volume production since it requires the design and creation of application specific tasks, which are rarely bug-free in the first instance. In addition, Matlab can be used to validate the studied algorithms in software and can easily be changed to an HDL language that can be used to build an FPGA.

\subsection{Low power consumption and low complexity video compression over wireless Zigbee net- works}

It is essential to use video compression techniques before transmitting video over a network especially for low bit-rate networks such as wireless Zigbee networks. Therefore, an MPEG4 encoder was used as our encoding standard since it is well known and works well over wireless networks. In addition, the transmission power will be decreased because the number of bits transmitted is reduced. It was also shown that with the introduction of region-of-interest coding and then passing the video sequence to the MPEG4 encoder, the video frame sizes are reduced substantially and hence the topology of the network increases. However, this video compression design still poses major challenges such as power, complexity, quality and delay issues. This is because both the video segmentation algorithm (ROI) and MPEG4 compression 
consume a high amount of energy that is not feasible for low power networks such as wireless Zigbee networks. In particular, in a typical video surveillance application, it has been shown that video encoding is mandatory due to the limited bandwidth and delay constraints. Whereas the compression itself (including motion compensation and frame differencing) can dominate the energy cost due to the high computational complexity of low power processors. The major power consumption in MPEG4 compression mainly occurs when calculating the P-frames and B-frames. This is because differences between the current frame and the reference frame require high computational complexity, which consumes high power and energy. Therefore, interesting future research would be to develop a video compression system that is mainly used for video surveillance over a wireless Zigbee network. The proposed algorithm should reduce the correlation between the current frame and its reference frame while at the same time keep the compression system close to the performance of the MPEG4 compression standard. In addition, the Zigbee node can be equipped a passive InfraRed sensor (PIR) for capturing images. To achieve low-power consumption, the PIR sensor detects an object regularly while the camera sensor is in its idle state. If there is an object, the node captures the video object with its camera. The use of PIR sensors would eliminate a high portion of the cost because the computations are only performed during objects appearances.

Figure 7.1 shows an example of a suitable encoder where the motion compensation and power consumption are eliminated by edge detection due to the fact that the video application will have low motion. PIR sensors are added to the compression technique so that the computations are only done when necessary. 


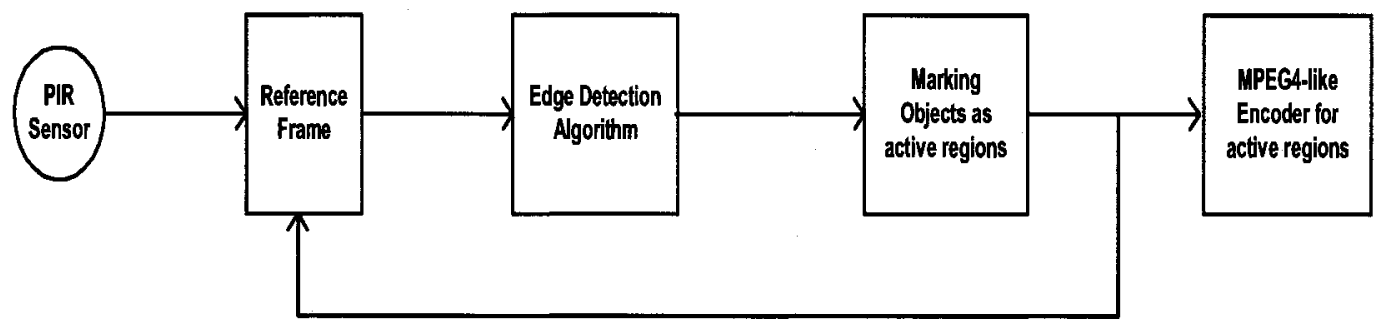

Figure 7.1: Simple compression algorithm for wireless surveillance Zigbee networks

\subsection{Scheduler design of multi-channel multi- interface topology}

It was shown in section 6.5 that the capacity of the wireless Zigbee network can be substantially increased by equipping each network node with multiple radio interfaces that can operate on multiple non-overlapping channels. This is motivated in IEEE 802.15.4 where the entire frequency band is divided into multiple channels, and each radio can only access one channel at a time. Hence, if each network node has multiple radio interfaces, it can then utilize a larger amount of radio bandwidth, and hence achieve a higher system capacity. Even if each node still has only one radio interface, when operating neighboring nodes at different channels, the amount of interference is reduced, which also leads to higher system capacity. However, all channel assignments that were used in figures $6.12,6.13$, and 6.14 are static, i.e. the channels were assigned before performing the simulations. The result of the static channel assignment is that no actions can be taken in case of interference or change in the channel conditions. 
Therefore, in order to fully utilize the increased bandwidth in multi-channel multiradio Zigbee networks, a set of resource allocation problems should be taken into consideration. These resource allocation problems include:

- channel assignment: The set of channels that each node/link should operate on.

- scheduling: The time when each link should be activated at a specific channel.

- routing: selecting the appropriate paths that minimize interference and increase throughput.

These three problems are inter-related with each other, and thus form a challenging cross-layer control problem across different layers. An interesting future development is to design a control protocol for multi-channel multi-radio Zigbee ad hoc networks that achieves high system capacity. Although some control protocols for channel assignment, scheduling and routing have been proposed in the literature, the algorithms do not take into account the computational complexity that arises with them. Hence, an efficient channel distributed assignment should be designed specifically for IEEE 802.15.4 multi-channel multi-radio networks. The channel assignment should consume the least power possible and try to adapt to various network conditions such as the appearance of interfering nodes or a change in the channel condition. The distributed approach is more efficient than the centralized one since it is less energy and time consuming. An example of an 802.15.4 node scheduler is shown in figure 7.2 .

\subsection{Enhanced channel modeling for simulations}

The wireless channel of the Zigbee network modeled in section 6.6 used a two-state Gilbert-Elliott channel model to include the bursty errors that occur in wireless channels. The channel can be modeled using a Finite-State Markov Channel (FSMC) following [61]. This is done by partitioning the signal to noise ratio (SNR) into a 


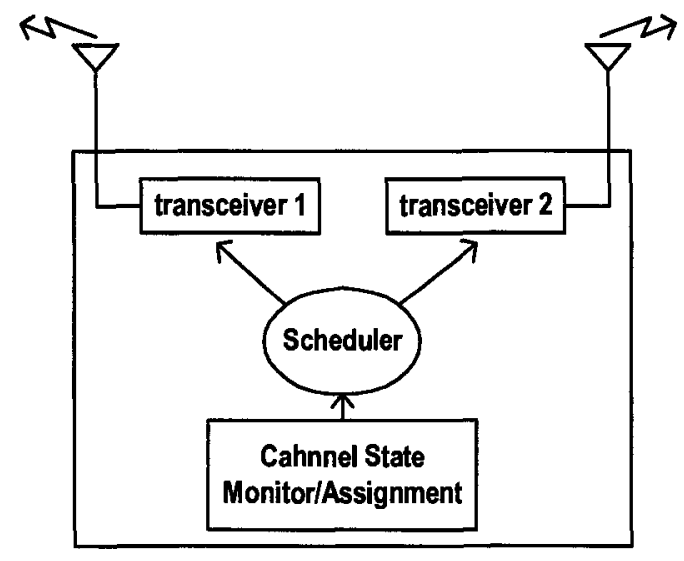

Figure 7.2: Scheduler of a multi-channel multi-interface 802.15 .4 node

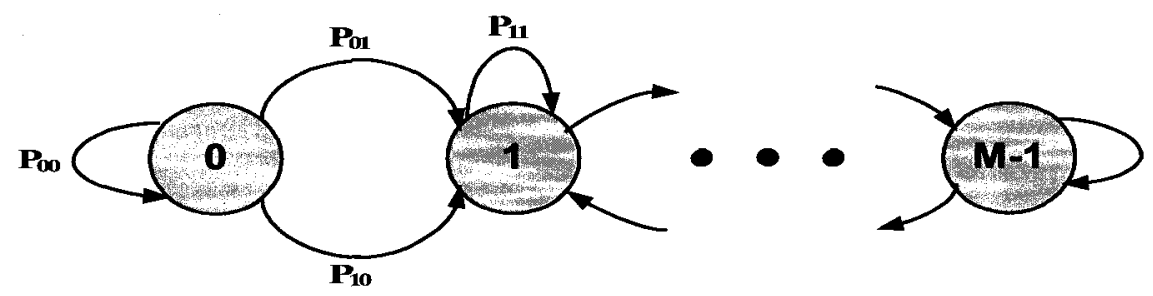

Figure 7.3: FSMC model for IEEE 802.15.4 channels

finite number of intervals, each representing a state in a Markov Chain. Assuming that the fading is slow enough, meaning that the channel states for consecutive time periods are neighboring states, then the model will be reduced into a discrete time birth and death process, as shown in figure 7.3.

Depending on the expected SNR, different modulation and data rates can be dynamically adapted to the rate control algorithm developed in this thesis. As data losses increase in the network, transmission rates will decrease. The SNR can even be mapped directly into a quantization parameter and hence into corresponding data 
rates. In light of this, the states in the channel model will equivalently represent data rate levels rather than SNR. 


\section{List of References}

[1] IEEE std. 802.15.4 - 2003: "Wireless Medium Access Control (MAC) and Physical Layer (PHY) specifications for Low Rate Wireless Personal Area Networks (LR-WPANs)"

[2] B. Melamed, D. Raychaudhuri, B. Sengupta and J. Zdepski, "TES-Based Traffic Modeling for Performance Evaluation of Integrated Networks", Proceedings of INFOCOM '92, Vol. 1, 75-84, Florence, Italy, 1992.

[3] K.W. Fendick, V.R. Saksena and W. Whitt, "Dependence in Packet Queues", IEEE Trans. on Comm., 37:1173-1183, 1989.

[4] M. Livny, B. Melamed and A.K. Tsiolis, "The Impact of Autocorrelation on Queuing Systems", Management Science, 1993.

[5] B. Melamed, "TES: A class of methods of generating autocorrelated uniform variates", ORSA Journal on Computing, vol. 3, no.4, pp.317-329, 1991.

[6] Elwalid, A.I. and Mitra, D. "'Effective Bandwidth of General Markovian Traffic Sources and dmission Control of High Speed Networks," IEEE/ACMTransactions on Networking, Vol. 1, No. 3, 1993.

[7] Frater, M., Tan, P. and Arnold, J. "Variable Bit Rate Video Traffic and the Broadband ISDN: Modeling and Verification", Proc. of the 14-th International Teletraffic Congress, June 1994, Antibes, France.

[8] Heyman, D., Tabatabai, A., Lakshman, T.V. and Heeke, H. "Modeling Teleconference Traffic from VBR Video Coders". Procedings of ICC '94, 1994.

[9] D. Geist and B. Melamed, "TEStool: An Environment forVisual Interactive Modeling of Autocorrelated Traffic", Proceedings of ICC 92, Vol. 3, 1285-1289, 1992, Chicago, Illinois.

[10] Zigbee Alliance website, http://www.zigbee.org 
[11] Y. Cao, Z. Ji, Z. Hu, "An image sensor node for wireless sensor networks," in Proc. International Conference on Information Technology: Coding and Computing (ITCC 2005), Apr. 2005, vol. 2, pp. 740-745.

[12] M. Rahimi, R. Baer, O. Iroezi, J. Garcia, J. Warrior, D. Estrin, M. Srivastava, "Cyclops: in situ image sensing and interpretation in wireless sensor networks", in: Proc. of the ACM Conf. on Embedded Networked Sensor Systems (SenSys), San Diego, CA, November 2005.

[13] Crossbow, http://www.xbow.com

[14] F. Oliveira, P. Chalimbaud, F. Berry, J. Serot, F. Marmoiton, "Embedded Early Vision Systems: Implementation Proposal and Hardware Architecture," in Proc. COGnitive systems with Interactive Sensors (COGIS 2006), Mar. 2006.

[15] I. Downes, L. Baghaei Rad, H. Aghajan, "Development of a Mote for Wireless Image Sensor Networks," in Proc. COGnitive systems with Interactive Sensors (COGIS 2006), Mar. 2006.

[16] S. Hengstler and H. Aghajan., "A smart camera mote architecture for distributed intelligent surveillance", In ACM SenSys Workshop on Distributed Smart Cameras (DSC), Boulder, Colorado, USA, October 2006

[17] Lam N., Mehrpour H, "TES modeling and multiplexing of MPEG video on ATM network", Proceedings of the International Symposium on Voice, Video and Data Communications, SPIE, The International Symposium on Voice, Video and Data Communications, Boston, Massachusetts, Nov. 1-5, 1998.

[18] Melamed, J. R. Hill, "A Survey of TES Modeling Applications", SIMULATION, Vol. 64, No. 6, 353 - 370, 1995.

[19] D.-S. Lee, B. Melamed, A. Reibman and B. Sengupta, "Analysis of a Video Multiplexer using TES as a Modeling Methodology", IEEE International Globecom 1991

[20] Matrawy, A. Lambadaris, L.Changcheng Huang, "MPEG4 traffic modeling using the transform expand sample methodology, "Networked Appliances. IEEE 4th International Workshop on Network Appliances, 2002.

[21] Sachin Deshpande, "Adaptive low-bitrate streaming over IEEE 802.15.4 low rate wireless personal area networks (LR-WPAN) based on link quality indication," International Conference On Communications And Mobile Computing, pages: $863-868,2006$ 
[22] D. Jagerman and B. Melamed, "The Transition and Autocorrelation Structure of TES Processes Part I: General Theory. Stochastic Models", Vol. 8, No. 2, 193-219, 1992.

[23] D. Jagerman and B. Melamed, "The Transition and Autocorrelation Structure of TES Processes Part II: Special Cases. Stochastic Models", Vol. 8, No. 3, 499-527, 1992.

[24] P.R. Jelenkovic and B. Melamed, "Automated TES modeling of compressed video", IEEE Trans. on Comms., vol. 2, pp.'746-752, 1995.

[25] ISO/IEC/JTC1/SC29/WG11, "Overview of the MPEG-4 standard" July 2000

[26] The Network Simulator - NS2 website, http://www.isi.edu/nsnam/ns/

[27] Abhinav Gupta, Ian Wormsbecker, Carey Williamson, "Experimental Evaluation of TCP Performance in Multi-Hop Wireless Ad Hoc Networks", mascots, pp. 311, 12th IEEE International Symposium on Modeling, Analysis, and Simulation of Computer and Telecommunications Systems (MASCOTS’04), 2004.

[28] "Coded Representation of Picture and Audio Information", ISOIEC/JTC1/SC29/WG11, MPEG Test Model 2, July 1992

[29] L. Galluccio, F. Licandro, G. Morabito, G. Schembra, "An Analytical Framework for the Design of Intelligent Algorithms for Adaptive-Rate MPEG Video Encoding in Next Generation Time-varying Wireless Networks", IEEE Journal on Selected Areas in Communications: Special Issue on Intelligent Services and Applications in Next Generation Networks, Vol. 23, No. 2, February 2005

[30] H. Gharavi and K. Ban, "Cross-layer feedback control for video communications via mobile ad-hoc networks," in Proc. 58th IEEE Vehicular Technology Conference (VTC '03), vol. 5, pp. 2941-2945, Orlando, Fla, USA, October 2003

[31] Feng Pan, Zhengguo Li, Keng Pang Lim, Xiao Lin, Susanto Rahardja, Si Wu, Dajun $\mathrm{Wu}$, "Adaptive intra-frame quantization for very low bit rate video coding," ISCAS (3) 2004: 781-784

[32] H. Liu and M. El Zarki, "Adaptive source rate control for real-time wireless video transmission," Mobile Networks and Applications. vol.3, no.1; pp.49-60, 1998

[33] S. Aramvith, I.-M. Pao, and M.-T. Sun, "A rate-control scheme for video transport over wireless channels," IEEE Transaction on Circuits and Systems for Video Technology, vol. 11, pp. 569-580, May. 2001 
[34] M. Hamdi, J. W. Roberts, P. Rolin, "Rate control for VBR video coders in broadband networks", IEEE Journal on Selected Areas in Communications, Vol. 15 no. 6,1997

[35] Hata, T., Kuwahara, N., Nozawa, T., Schewenke, D.L., Vetro, A, "Surveillance system with object-aware video transcoder" Proc. Multimedia Signal Processing, (MMSP-05), Shanghai, China, 2005

[36] P. Baccichet, X. Zhu, and B. Girod, "Network-aware h.264/avc region-of-interest coding for a multi-camera wireless surveillance network," April 2006.

[37] D. Zhong and S.-F. Chang, "Long-Term Moving Object Segmentation and Tracking Using Spatio-Temporal Consistency", IEEE International Conference on Image Processing, Greece, Oct. 2001.

[38] J. Klaue, B. Rathke, and A. Wolisz, "EvalVid - A Framework for Video Transmission and Quality Evaluation", International Conference on Modelling Techniques and Tools for Computer Performance Evaluation, pp. 255-272, USA, September 2003.

[39] I. Howitt and J.A. Gutierrez, "IEEE 802.15.4 low rate - wireless personal area network coexistence issues," IEEE Wireless Communications and Networking, vol. 3, pp. 1481 - 1486, 2003.

[40] S. Shin, S. Choi, H. S. Park, and W. H. Kwon, "Packet error rate analysis of IEEE 802.15.4 under IEEE 802.11b interference," in Proceedings of WWIC 2005, LNCS, Springer, May, pp. 279-288.

[41] N. Golmie, D. Cypher, O. Rebala, "Performance Analysis of Low Rate Wireless Technologies for Medical Applications," Computer Communications, Volume 28, Number 10, June 2005, pages 1255-1275

[42] C. Won, et al., "Adaptive Radio Channel Allocation for Supporting Coexistence of 802.15.4 and 802.11b," in Proc. VTC, Vol. 4, pp. 2522 - 2526, Sep 2005.

[43] S. Pollin, et al., "Distributed cognitive coexistence of 802.15 .4 with 802.11," in Proc. Crowncom, 2006.

[44] Min Suk Kang, Jo Woon Chong, Hyesun Hyun, Su Min Kim, Byoung Hoon Jung, and Dan Keun Sung "Adaptive Interference-Aware Multi-Channel Clustering Algorithm in a ZigBee Network in the Presence of WLAN Interference," ISWPC, 2007. 
[45] Y. Liu, E. Knightly, "Opportunistic Fair Scheduling over Multiple Wireless Channels", Proc. of IEEE INFOCOM 2003.

[46] J. So, N. Vaidya, "Multi-Channel MAC for Ad Hoc Networks: Handling MultiChannel Hidden Terminals Using A Single Transceiver", Proc. of MobiHOC 2004.

[47] Paramvir Bahl, Ranveer Chandra, and John Dunagan, "SSCH: Slotted Seeded Channel Hopping for Capacity Improvement in IEEE 802.11 Ad-Hoc Wireless Networks," in ACM Mobicom, 2004.

[48] Shih-Lin Wu, Chih-Yu Lin, Yu-Chee Tseng, and Jang-Ping Sheu, "A New MultiChannel MAC Protocol with On-Demand Channel Assignment for Multi-Hop Mobile Ad Hoc Networks," in International Symposium on Parallel Architectures, Algorithms and Networks (ISPAN), 2000.

[49] R. Draves, J. Padhye, and B. Zill, "Routing in Multi-radio, Multi-hop Wireless Mesh Networks", ACM MobiCom 2004.

[50] R. Karrer, A. Sabharwal, E. Knightly, "Enabling Large-scale Wireless Broadband: The Case for TAPs", Proc. of the HotNets 2003

[51] Ashish Raniwala, Kartik Gopalan, and Tzi-cker Chiueh, "Centralized Channel Assignment and Routing Algorithms for Multi-Channel Wireless Mesh Networks," Mobile Computing and Communications Review, vol. 8, no. 2, pp. 50-65, April 2004.

[52] Ashish Raniwala and Tzi-cker Chiueh, "Architecture and Algorithms for an IEEE 802.11-Based Multi-Channel Wireless Mesh Network," in Infocom, 2005

[53] The Enhanced Network Simulator. http://www.cse.iitk.ac.in/users/braman/tens.

[54] Laurent Paquereau and Bjarne E. Helvik, "A module-based wireless node for ns-2", in Proceedings of the first Workshop on NS2: the IP network simulator (WNS2), Pisa, Italy, 2006.

[55] J. Apostolopoulos, W.Tan, S. Wee and G.W. Wornell, "Modeling Path Diversity for Multiple Description Video Communication", IEEE Intern Conf. Acoustics, Speech and Signal Processing, 2002

[56] Lallet, A. Dolbear, C. Hughes, J. Hobson, P, "Review of scalable video strategies for distributed video applications ", Distributed Imaging (Ref. No. 1999/109), IEE European Workshop, 1999 
[57] R. Singh, A Ortega, L.Perret and W.Jiang, "Comparison of Multiple Description Coding and Layered Coding Based on Network Simulation," VCIP 2000

[58] Y. Wang, S. Panwar, S. Lin, and S. Mao, "Wireless Video Transport Using Path Diversity: Multiple Description vs. Layered Coding," Image Processing. 2002. Proceedings. 2002 International Conference on, Volume: 1, 2002

[59] Frank H.P. Fitzek, B. Can, R. Prasad, and M. Katz, "Overhead and Quality Measurements for Multiple Description Coding for Video Services", Wireless. Personal Multimedia Communications (WPMC), September 2004

[60] Changhua He, Jinhui Pan, "Comparing Layered Coding and Multiple Description Coding with Link Diversity", Stanford University, 2005

[61] H. S. Wang and N. Moayeri, "Finite-State Markov Channel-A UsefulModel for Radio Communication Channels", IEEE Transactions on Vehicular Technology, vol. 44, pp. 163171, Feb. 1995. 